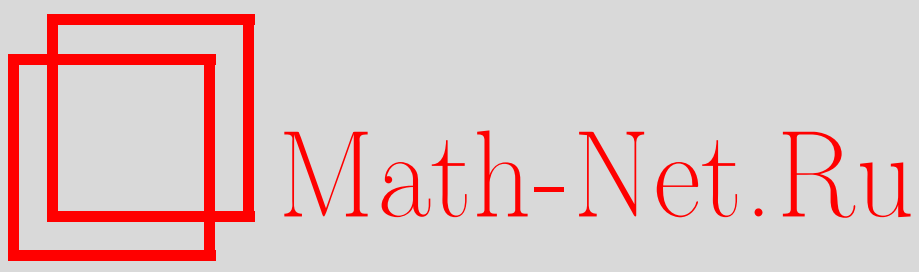

Л. И. Данилов, Мерозначные почти периодические функции и почти периодические сечения многозначных отображений, Матем. сб., 1997, том 188, номер 10, 3-24

DOI: https://doi.org/10.4213/sm262

Использование Общероссийского математического портала Math-Net.Ru подразумевает, что вы прочитали и согласны с пользовательским соглашением http://www . mathnet.ru/rus/agreement

Параметры загрузки:

IP: 3.91 .87 .62

26 апреля 2023 г., $17: 36: 46$ 
УДК 517.9

\author{
Л.И. Данилов
}

\title{
Мерозначные почти периодические функции и почти периодические сечения многозначных отображений
}

\begin{abstract}
В работе изучаются почти периодические по Степанову сечения многозначных отображений $t \mapsto \operatorname{supp} \mu[\cdot ; t], t \in \mathbb{R}$. Мера $\mu[\cdot ; t]$ при п.в. $t \in \mathbb{R}$ предполагается вероятностной мерой Радона на полном метрическом пространстве и $t \mapsto \operatorname{supp} \mu[\cdot ; t], t \in \mathbb{R},-$ мерозначная почти периодическая функция.

Библиография: 17 названий.
\end{abstract}

Пусть $(\mathscr{U}, \rho)$ - полное метрическое пространство, $\bar{A}$ - замыкание множества $A \subset \mathscr{U}, \rho(x, A)=\inf _{y \in A} \rho(x, y)$ - расстояние от точки $x \in \mathscr{U}$ до непустого множества $A \subset \mathscr{U},(\mathrm{cl} \mathscr{U}$, dist $)$ - метрическое пространство непустых замкнутых ограниченных множеств $A \subset \mathscr{U}$ с метрикой Хаусдорфа

$$
\operatorname{dist}(A, B)=\max \left\{\sup _{x \in A} \rho(x, B), \sup _{x \in B} \rho(x, A)\right\}, \quad A, B \in \operatorname{cl} \mathscr{U} .
$$

Известно, что у почти периодических (п.п.) по Бору (равномерных п.п.) многозначных отображений $t \mapsto F(t) \in(\mathrm{cl} \mathscr{U}$, dist $), t \in \mathbb{R}$, может не существовать п.п. по Бору сечений. В частности, в конечномерном евклидовом пространстве не всегда можно п.п. по Бору ортогональный репер дополнить до п.п. по Бору ортогонального базиса [1]. Последнее означает, что не всегда существуют п.п. по Бору сечения у многозначного п.п. по Бору отображения, образы которого состоят из точек единичной сферы, ортогональных п.п. реперу. Несложно также построить пример не почти периодической по Бору (и даже не почти периодической по Степанову) непрерывной функции $t \mapsto f(t) \in \mathbb{R}^{2} \backslash\{0\}, t \in \mathbb{R}$, такой, что $t \mapsto\{f(t),-f(t)\} \in \mathrm{cl} \mathbb{R}^{2}$, $t \in \mathbb{R},-$ двузначное п.п. по Бору отображение [2]. Другая ситуация возникает при рассмотрении сечений, которые почти периодичны по Степанову. В [3] в случае сепарабельного банахова пространства $\mathscr{U}$ было доказано, что у любого многозначного п.п. по Степанову (степени 1 ) отображения $t \mapsto F(t) \in \mathrm{cl} \mathscr{U}, t \in \mathbb{R}$, существует счетное множество п.п. по Степанову (степени 1 ) сечений $f_{j} \in S_{1}(\mathbb{R}, \mathscr{U}), j \in \mathbb{N}$, таких, что $\operatorname{Mod} f_{j} \subset \operatorname{Mod} F$ и замыкание множества $\bigcup_{j} f_{j}(t)$ при почти всех (п.в.) $t \in \mathbb{R}$ совпадает с $F(t)$.

В силу теоремы Кастена [4, теорема III], [5, с. 331], [6]-[9] многозначное отображение $t \mapsto F(t) \subset \mathscr{U}, t \in \mathbb{R}$, с непустыми замкнутыми образами в полном сепарабельном метрическом пространстве $\mathscr{U}$ можно представить в виде $F(t)=$ $\overline{\bigcup_{j} f_{j}(t)}$ п.в., где $f_{j}: \mathbb{R} \rightarrow \mathscr{U}, j \in \mathbb{N},-$ измеримые функции, тогда и только тогда, когда оно измеримо, т.е. для любого открытого множества $\mathscr{O} \subset \mathscr{U}$ множество $\{t \in \mathbb{R}: F(t) \cap \mathscr{O} \neq \varnothing\}$ измеримо (по Лебегу). Если измеримые функции 
$f_{j}, j \in \mathbb{N}$, принадлежат некоторому классу функций (или обладают какими-либо дополнительными свойствами), то возникает задача охарактеризовать многозначные отображения $t \mapsto F(t) \subset \mathscr{U}, t \in \mathbb{R}$, которые при п.в. $t \in \mathbb{R}$ представимы в виде $F(t)=\overline{\bigcup_{j} f_{j}(t)}$, где $f_{j}$ - функции из рассматриваемого класса. В настояшей работе рассматриваются сечения $f_{j}$ из классов $S(\mathbb{R}, \mathscr{U}), S_{p}(\mathbb{R}, \mathscr{U}), p \geqslant 1$, и $S^{c}(\mathbb{R}, \mathscr{U})$ п.п. по Степанову функций (функции $f \in S^{c}(\mathbb{R}, \mathscr{U})$ п.в. принимают значения из компактных множеств $K(f) \subset \mathscr{U})$. Для полного метрического пространства $\mathscr{U}$ доказано, что многозначное отображение $t \mapsto F(t) \subset \mathscr{U}, t \in \mathbb{R}$, можно представить в виде $F(t)=\overline{\bigcup_{j} f_{j}(t)}$ п.в., где $f_{j} \in S(\mathbb{R}, \mathscr{U}), j \in \mathbb{N}$, тогда и только тогда, когда множество $F(t)$ при п.в. $t \in \mathbb{R}$ является носителем supp $\mu[\cdot ; t]$ мерозначной п.п. по Степанову функции $t \mapsto \mu[\cdot ; t], t \in \mathbb{R}$, со значениями в пространстве вероятностных мер Радона. При этом сечения $f_{j} \in S(\mathbb{R}, \mathscr{U}), j \in \mathbb{N}$, многозначного отображения $t \mapsto \operatorname{supp} \mu[\cdot ; t], t \in \mathbb{R}$, можно выбрать так, чтобы меры $\mu[\cdot ; t]$ аппроксимировались линейными комбинациями мер Дирака

$$
\frac{1}{N} \sum_{j=1}^{N} \delta_{f_{j}(t)}[\cdot], \quad N \rightarrow \infty, \quad t \in \mathbb{R}
$$

Утверждения о п.п. по Степанову сечениях многозначных отображений имеют ряд особенностей, которые отличают их от аналогичных утверждений для измеримых сечений. Пусть $\mathrm{cl}_{n} \mathscr{U}$ - совокупность множеств $A \in \mathrm{cl} \mathscr{U}$, состоящих не более чем из $n$ точек, $n \in \mathbb{N}$. Если $t \mapsto F(t) \in \mathrm{cl}_{n} \mathscr{U}, t \in \mathbb{R},-$ п.п. по Степанову многозначное отображение, то для любого $\varepsilon>0$ найдутся функции $f_{j} \in S(\mathbb{R}, \mathscr{U})$, $j=1, \ldots, n$, такие, что $\operatorname{Mod} f_{j} \subset \operatorname{Mod} F, f_{j}(t) \in F(t)$ п.в. и $\operatorname{dist}\left(F(t), \bigcup_{j} f_{j}(t)\right)<\varepsilon$ п.в. [6]. Однако не всегда можно найти $n$ функций $f_{j} \in S(\mathbb{R}, \mathscr{U}), j=1, \ldots, n$, для которых $F(t)=\bigcup_{j} f_{j}(t)$ п.в. Более того, в [2] для всех $n \in \mathbb{N}$ приведены примеры $n$-значных п.п. по Степанову отображений $t \mapsto F_{n}(t) \in \operatorname{cl}_{n}[-1,1], t \in \mathbb{R}$, для которых равенство $F_{n}(t)=\bigcup_{j} f_{j}(t)$ п.в. не может выполняться ни для каких функций $f_{j} \in S(\mathbb{R}, \mathbb{R}), j=1, \ldots, 2^{n-1}-1$. В [2], [6] доказано, что для любого п.п. по Степанову многозначного отображения $t \mapsto F(t) \in \operatorname{cl}_{n} \mathscr{U}, t \in \mathbb{R}$, $n \in \mathbb{N}$, всегда найдется $2^{n-1}$ сечений $f_{j} \in S(\mathbb{R}, \mathscr{U})$, для которых $F(t)=\bigcup_{j} f_{j}(t)$ п.в. и $\operatorname{Mod} f_{j} \subset \operatorname{Mod} F, j=1, \ldots, 2^{n-1}\left(\right.$ если $F \in S_{p}\left(\mathbb{R}, \operatorname{cl}_{n} \mathscr{U}\right), p \geqslant 1$, то $\left.f_{j} \in S_{p}(\mathbb{R}, \mathscr{U})\right)$.

Ряд других утверждений о п.п. по Степанову сечениях многозначных отображений приведен в [2], [6]-[9]. При их доказательстве существенно применялась равномерная аппроксимация п.п. функций элементарными (принимающими счетное множество значений) п.п. по Степанову функциями [2], [6]. Эта же аппроксимация будет использоваться и в настоящей работе.

В $\S 1$ приведены определения и некоторые утверждения о п.п. функциях со значениями в метрических пространствах, а также о вероятностных борелевских меpax, которые постоянно используются в дальнейшем. Большинство стандартных утверждений о п.п. функциях содержится в [10], [11], относительно вероятностных борелевских мер см. [12]. Один из вариантов теоремы о равномерной аппроксимации п.п. функций доказывается в $\S 2$. В $\S 3$ сформулированы основные результаты работы. В последующих параграфах доказываются теоремы из $\S 3$. 


\section{§1. Определения, обозначения и некоторые утверждения}

Пусть $(\mathscr{U}, \rho)$ - полное метрическое пространство,

$$
K(x, r)=\{y \in \mathscr{U}: \rho(x, y) \leqslant r\}, \quad x \in \mathscr{U}, \quad r \geqslant 0
$$

Множество $K \subset \mathscr{U}$ предкомпактно, если $\bar{K}$ - компактное множество.

Функция $f: \mathbb{R} \rightarrow \mathscr{U}$ называется простой [12], если она принимает конечное число значений на измеримых (по Лебегу) множествах. Функция $f: \mathbb{R} \rightarrow \mathscr{U}$ измеримa, если она п.в. совпадает с пределом п.в. сходящейся последовательности простых функций. Класс измеримых функций обозначим через $M(\mathbb{R}, \mathscr{U})$ (функции, совпадающие при п.в. $t \in \mathbb{R}$, отождествляются). Для функций $f, g \in M(\mathbb{R}, \mathscr{U})$ и $p \geqslant 1$ положим

$$
\begin{aligned}
& D_{\infty}^{(\rho)}(f, g)=\operatorname{essip}_{t \in \mathbb{R}} \rho(f(t), g(t)), \\
& D_{p}^{(\rho)}(f, g)=\sup _{\xi \in \mathbb{R}}\left(\int_{\xi}^{\xi+1} \rho^{p}(f(t), g(t)) d t\right)^{1 / p} .
\end{aligned}
$$

Зафиксируем элемент $x_{0} \in \mathscr{U}$. Обозначим

$$
\begin{aligned}
L^{\infty}(\mathbb{R}, \mathscr{U}) & =\left\{f \in M(\mathbb{R}, \mathscr{U}): D_{\infty}^{(\rho)}\left(f(\cdot), x_{0}(\cdot)\right)<\infty\right\}, \\
M_{p}(\mathbb{R}, \mathscr{U}) & =\left\{f \in M(\mathbb{R}, \mathscr{U}): D_{p}^{(\rho)}\left(f(\cdot), x_{0}(\cdot)\right)<\infty\right\}, \quad p \geqslant 1,
\end{aligned}
$$

где $x_{0}(t) \equiv x_{0}, t \in \mathbb{R}$. Функции $f \in M_{p}(\mathbb{R}, \mathscr{U})$, для которых

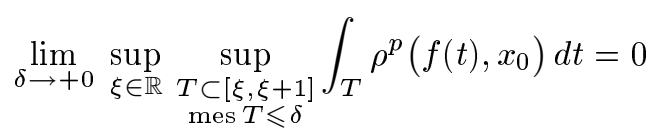

(mes - мера Лебега), образуют замкнутое подмножество $M_{p}^{\prime}(\mathbb{R}, \mathscr{U})$ полного метрического пространства $\left(M_{p}(\mathbb{R}, \mathscr{U}), D_{p}^{(\rho)}\right)$. Ни $M_{p}(\mathbb{R}, \mathscr{U})$, ни $M_{p}^{\prime}(\mathbb{R}, \mathscr{U})$ не зависят от выбора элемента $x_{0}$.

Число $\tau \in \mathbb{R}$ называется $\varepsilon$-почти периодом функиии $f \in M_{p}(\mathbb{R}, \mathscr{U})$, если $D_{p}^{(\rho)}(f(\cdot+\tau), f(\cdot))<\varepsilon$. Функция $f: \mathbb{R} \rightarrow \mathscr{U}$ принадлежит множеству $S_{p}(\mathbb{R}, \mathscr{U})$ почти периодических по Степанову функций степени $p \geqslant 1$, если $f \in M_{p}(\mathbb{R}, \mathscr{U})$ и для любого $\varepsilon>0$ множество $\varepsilon$-почти периодов функции $f$ относительно плотно [10]. При этом $S_{p}(\mathbb{R}, \mathscr{U})$ - замкнутое подмножество множества $M_{p}^{\prime}(\mathbb{R}, \mathscr{U}) \subset$ $\left(M_{p}(\mathbb{R}, \mathscr{U}), D_{p}^{(\rho)}\right)$.

Последовательность $\left\{\tau_{j}\right\} \subset \mathbb{R}$ назьвается $f$-возвращающей для функции $f \in$ $S_{p}(\mathbb{R}, \mathscr{U})$, если $\left.D_{p}^{(\rho)}\left(f \cdot+\tau_{j}\right), f(\cdot)\right) \rightarrow 0$ при $j \rightarrow \infty$. Для каждой функции $f \in S_{p}(\mathbb{R}, \mathscr{U})$ определяется множество $\operatorname{Mod} f$, состоящее из тех чисел $\lambda \in \mathbb{R}$, для которых $e^{i \lambda \tau_{j}} \rightarrow 1$ при $j \rightarrow \infty$ для любой $f$-возвращающей последовательности $\left\{\tau_{j}\right\}$. Если функция $f \in S_{p}(\mathbb{R}, \mathscr{U})$ не является (п.в.) постоянной, то $\operatorname{Mod} f-$ счетное множество. В случае банахова пространства $\mathscr{U}$ множество Mod $f$ совпадает с модулем показателей Фурье функции $f \in S_{p}(\mathbb{R}, \mathscr{U})$. 
Функция $f \in M(\mathbb{R}, \mathscr{U})$ принадлежит пространству $S(\mathbb{R}, \mathscr{U})$ почти периодических по Степанову функций, если для любых $\varepsilon, \delta>0$ множество чисел $\tau \in \mathbb{R}$, для которых

$$
\sup _{\xi \in \mathbb{R}} \operatorname{mes}\{t \in[\xi, \xi+1]: \rho(f(t+\tau), f(t)) \geqslant \varepsilon\}<\delta,
$$

относительно плотно. Последовательность $\left\{\tau_{j}\right\} \subset \mathbb{R}$ называется $f$-возвращающей для функции $f \in S(\mathbb{R}, \mathscr{U})$, если для любого $\varepsilon>0$

$$
\lim _{j \rightarrow \infty} \sup _{\xi \in \mathbb{R}} \operatorname{mes}\left\{t \in[\xi, \xi+1]: \rho\left(f\left(t+\tau_{j}\right), f(t)\right) \geqslant \varepsilon\right\}=0 .
$$

С помощью $f$-возвращающих последовательностей $\left\{\tau_{j}\right\}$ для любой функции $f \in S(\mathbb{R}, \mathscr{U})$ (аналогично функциям из пространств $S_{p}(\mathbb{R}, \mathscr{U}), p \geqslant 1$ ) определяется $\operatorname{Mod} f$.

Определим функцию $\theta: \theta(\xi)=0$ при $\xi \leqslant 0, \theta(\xi)=\xi$ при $0 \leqslant \xi \leqslant 1$ и $\theta(\xi)=1$ при $\xi \geqslant 1$. Введем метрику $\rho^{\prime}(x, y)=\theta(\rho(x, y)), x, y \in \mathscr{U}$. Функция $f: \mathbb{R} \rightarrow \mathscr{U}$ принадлежит пространству $S(\mathbb{R},(\mathscr{U}, \rho))$ тогда и только тогда, когда $f \in S_{1}\left(\mathbb{R},\left(\mathscr{U}, \rho^{\prime}\right)\right)$. При этом $f$-возвращающие последовательности (и $\left.\operatorname{Mod} f\right)$ в пространствах $S(\mathbb{R},(\mathscr{U}, \rho))$ и $S_{1}\left(\mathbb{R},\left(\mathscr{U}, \rho^{\prime}\right)\right)$ одни и те же.

Если $f \in S_{p}(\mathbb{R}, \mathscr{U}), p \geqslant 1$, то $f \in S(\mathbb{R}, \mathscr{U})$ и $\operatorname{Mod} f$ не зависит от рассматриваемого пространства функций.

Для функций $f, g \in M(\mathbb{R}, \mathscr{U})$ положим $D^{(\rho)}(f, g)=D_{1}^{\left(\rho^{\prime}\right)}(f, g)$. Множество $S(\mathbb{R}, \mathscr{U})$ замкнуто в полном метрическом пространстве $\left(M(\mathbb{R}, \mathscr{U}), D^{(\rho)}\right)$.

Пусть $f, f_{j} \in S(\mathbb{R}, \mathscr{U}), j \in \mathbb{N}$, и $D^{(\rho)}\left(f, f_{j}\right) \rightarrow 0$ при $j \rightarrow \infty$. Тогда $\operatorname{Mod} f \subset$ $\sum \operatorname{Mod} f_{j}$ (справа стоит сумма модулей, т.е. наименьший модуль в $\mathbb{R}$ (группа по сложению), содержаший все множества $\left.\operatorname{Mod} f_{j}\right)$. Если $f \in S(\mathbb{R}, \mathscr{U}), f_{i} \in S\left(\mathbb{R}, \mathscr{U}_{i}\right)$, где $\mathscr{U}_{i}$ - произвольные метрические пространства, $i \in \mathbb{N}$, то $\operatorname{Mod} f \subset \sum \operatorname{Mod} f_{i}$

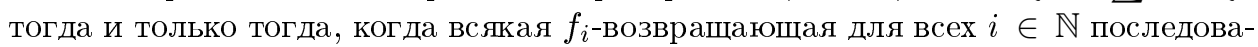
тельность $\left\{\tau_{j}\right\}$ является $f$-возвращающей.

Обозначим через $S^{c}(\mathbb{R}, \mathscr{U})$ множество функций $f \in S(\mathbb{R}, \mathscr{U})$, для которых существует компакт $K=K(f) \subset \mathscr{U}$ такой, что $f(t) \in K$ п.в. Если $f \in M(\mathbb{R}, \mathscr{U})$, $f_{j} \in S^{c}(\mathbb{R}, \mathscr{U}), j \in \mathbb{N}$, и $D_{\infty}^{(\rho)}\left(f, f_{j}\right) \rightarrow 0$ при $j \rightarrow \infty$, то $f \in S^{c}(\mathbb{R}, \mathscr{U})$.

Для любого $p \geqslant 1$ имеет место равенство

$$
S_{p}(\mathbb{R}, \mathscr{U})=S(\mathbb{R}, \mathscr{U}) \cap M_{p}^{\prime}(\mathbb{R}, \mathscr{U})
$$

и справедлива следующая простая лемма.

Лемма 1. Пусть $f, f_{j} \in S_{p}(\mathbb{R}, \mathscr{U}), j \in \mathbb{N}$. Тогда $D_{p}^{(\rho)}\left(f, f_{j}\right) \rightarrow 0$ nри $j \rightarrow \infty$ в том и только в том случае, если $D^{(\rho)}\left(f, f_{j}\right) \rightarrow 0$ при $j \rightarrow \infty u$

$$
\lim _{\delta \rightarrow+0} \sup _{j} \sup _{\xi \in \mathbb{R}} \sup _{\substack{T \subset[\xi, \xi+1] \\ \operatorname{mes} T \leqslant \delta}} \int_{T} \rho^{p}\left(f_{j}(t), x_{0}\right) d t=0 .
$$

В настоящей работе в основном будут рассматриваться сечения многозначных отображений из пространств $S^{c}(\mathbb{R}, \mathscr{U})$ и $S(\mathbb{R}, \mathscr{U})$. Равенство (1) и лемма 1 позволяют использовать результаты работы при получении утверждений о сечениях, принадлежаших пространствам $S_{p}(\mathbb{R}, \mathscr{U}), p \geqslant 1$. 
Через $S(\mathbb{R})$ будем обозначать совокупность измеримых множеств $T \subset \mathbb{R}$ таких, что $\chi_{T}(\cdot) \in S_{1}(\mathbb{R}, \mathbb{R})\left(\chi_{T}(\cdot)\right.$ - характеристическая функция множества $\left.T\right)$. Для множества $T \in S(\mathbb{R})$ положим $\operatorname{Mod} T=\operatorname{Mod} \chi_{T}$. Для любого измеримого множества $T \subset \mathbb{R}$ обозначим

$$
\varkappa(T)=\sup _{\xi \in \mathbb{R}} \operatorname{mes}([\xi, \xi+1] \backslash T) .
$$

Если $T_{j} \in S(\mathbb{R}), j \in \mathbb{N}$, и $\sum_{j} \varkappa\left(T_{j}\right)<\infty$, то $\bigcap T_{j} \in S(\mathbb{R}), \operatorname{Mod} \bigcap T_{j} \subset \sum_{j} \operatorname{Mod} T_{j}$ и $\varkappa\left(\bigcap T_{j}\right) \leqslant \sum_{j} \varkappa\left(T_{j}\right)$.

Пусть $\Lambda$ - произвольный модуль (группа по сложению) в $\mathbb{R}$. Обозначим через $\mathfrak{M}(\Lambda)$ множество последовательностей $\left\{T_{j}\right\}, j \in \mathbb{N}$, непересекающихся множеств $T_{j} \in S(\mathbb{R})$ таких, что $\operatorname{Mod} T_{j} \subset \Lambda$ и $\lim _{N \rightarrow \infty} \varkappa\left(\bigcup_{j \leqslant N} T_{j}\right)=0$ (тогда $\left.\operatorname{mes}\left(\mathbb{R} \backslash \bigcup T_{j}\right)=0\right)$. Последовательности из $\mathfrak{M}(\Lambda)$ в дальнейшем будут нумероваться также с помошью нескольких индексов (и могут состоять из конечного числа множеств $T_{j}$, которые всегда можно дополнить пустыми множествами до счетной последовательности). Если $\left\{T_{i}^{(1)}\right\} \in \mathfrak{M}\left(\Lambda_{1}\right),\left\{T_{j}^{(2)}\right\} \in \mathfrak{M}\left(\Lambda_{2}\right)$, где $\Lambda_{1}$ и $\Lambda_{2}$ - произвольные модули в $\mathbb{R}$, то

$$
\lim _{N \rightarrow \infty} \varkappa\left(\bigcup_{i, j \leqslant N}\left(T_{i}^{(1)} \cap T_{j}^{(2)}\right)\right)=0,
$$

поэтому $\left\{T_{i}^{(1)} \cap T_{j}^{(2)}\right\} \in \mathfrak{M}\left(\Lambda_{1}+\Lambda_{2}\right)$.

Пусть $\left\{T_{j}\right\} \in \mathfrak{M}(\mathbb{R})$ и $f_{j} \in S(\mathbb{R}, \mathscr{U}), j \in \mathbb{N}$. Определим функцию $\sum f_{j}(\cdot) \chi_{T_{j}}(\cdot)$, совпадаюшую с функцией $f_{j}(\cdot)$ на множестве $T_{j}, j \in \mathbb{N}$. Функция $\sum f_{j}(\cdot) \chi_{T_{j}}(\cdot)$ определена почти всюду и измерима (используемое обозначение формально некорректно, но никаких линейных операций над рассматриваемьми функциями производиться не будет). В частности, если $x_{j} \in \mathscr{U}, j \in \mathbb{N}$, то через $\sum x_{j} \chi_{T_{j}}(\cdot)$ будет обозначаться элементарная функция, принимающая на множестве $T_{j}$ значение $x_{j}$, $j \in \mathbb{N}[12]$.

Лемма 2 [7]. Пусть $\left\{T_{j}\right\} \in \mathfrak{M}(\mathbb{R}) u f_{j} \in S(\mathbb{R}, \mathscr{U}), j \in \mathbb{N}$. Тогдa $\sum f_{j}(\cdot) \chi_{T_{j}}(\cdot)$ $\in S(\mathbb{R}, \mathscr{U}) u \operatorname{Mod} \sum f_{j}(\cdot) \chi_{T_{j}}(\cdot) \subset \sum_{j} \operatorname{Mod} f_{j}+\sum_{j} \operatorname{Mod} T_{j}$.

Для доказательства леммы 2 достаточно установить, что

$$
\begin{gathered}
g_{N}(\cdot) \doteq \sum_{j=1}^{N} f_{j}(\cdot) \chi_{T_{j}}(\cdot)+x_{0} \chi_{\mathbb{R} \backslash \bigcup_{j=1}^{N} T_{j}}(\cdot) \in S(\mathbb{R}, \mathscr{U}), \\
\operatorname{Mod} g_{N} \subset \sum_{j=1}^{N} \operatorname{Mod} f_{j}+\sum_{j=1}^{N} \operatorname{Mod} T_{j}, \quad N \in \mathbb{N}, \\
D^{(\rho)}\left(\sum f_{j}(\cdot) \chi_{T_{j}}(\cdot), g_{N}(\cdot)\right) \rightarrow 0, \quad N \rightarrow \infty .
\end{gathered}
$$

С помощью леммы 2 будут строиться почти периодические по Степанову сечения многозначных отображений. 
Для произвольного метрического пространства $(\mathscr{V}, \tilde{\rho})$ обозначим через $C(\mathscr{U}, \mathscr{V})$ множество непрерывных функций $\mathscr{F}: \mathscr{U} \rightarrow \mathscr{V}, C_{b}(\mathscr{U}, \mathscr{V})$ - метрическое пространство ограниченных функций $\mathscr{F} \in C(\mathscr{U}, \mathscr{V})$ с метрикой

$$
D_{C_{b}(\mathscr{U}, \mathscr{V})}(\mathscr{F}, G)=\sup _{x \in \mathscr{U}} \tilde{\rho}(\mathscr{F}(x), G(x)), \quad \mathscr{F}, G \in C_{b}(\mathscr{U}, \mathscr{V}) .
$$

Если $(\mathscr{V}, \tilde{\rho})=(\mathbb{R},|\cdot|)$, то обозначим $C(\mathscr{U}, \mathscr{V})=C(\mathscr{U})$ и $C_{b}(\mathscr{U}, \mathscr{V})=C_{b}(\mathscr{U})$, при этом $C_{b}(\mathscr{U})$ - банахово пространство с нормой

$$
\|\mathscr{F}\|=\|\mathscr{F}\|_{C_{b}(\mathscr{U})}=\sup _{x \in \mathscr{U}}|\mathscr{F}(x)|, \quad \mathscr{F} \in C_{b}(\mathscr{U}) ;
$$

$C_{b}^{+}(\mathscr{U})$ - множество неотрицательных функций из $C_{b}(\mathscr{U})$.

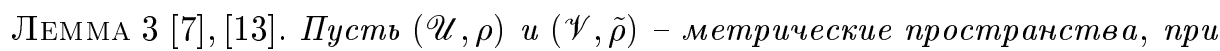
этом пространство $\mathscr{U}$ полное. Если $f \in S(\mathbb{R}, \mathscr{U})$ и функция $t \mapsto \mathscr{F}(\cdot, t) \in$ $C_{b}(\mathscr{U}, \mathscr{V}), t \in \mathbb{R}$, принадлежит пространству $S\left(\mathbb{R},\left(C_{b},(\mathscr{U}, \mathscr{V}), D_{C_{b}(\mathscr{U}, \mathscr{V})}\right)\right)$, mo $\mathscr{F}(f(\cdot), \cdot) \in S(\mathbb{R}, \mathscr{V})$ u $\operatorname{Mod} \mathscr{F}(f(\cdot), \cdot) \subset \operatorname{Mod} f+\operatorname{Mod} \mathscr{F}(\cdot, \cdot)$.

Приведем краткую схему доказательства леммы 3 . Пусть $(\widetilde{\mathcal{V}}, \tilde{\rho})$ - пополнение метрического пространства $(\mathscr{V}, \tilde{\rho})$. Функции $f$ и $\mathscr{F}(\cdot, \cdot)$ почти периодичны по Степанову, поэтому для любого $\delta>0$ найдутся измеримое множество $T_{\delta} \subset \mathbb{R}$ и компакты $K_{\delta} \subset \mathscr{U}, \mathscr{K}_{\delta} \subset C_{b}(\mathscr{U}, \widetilde{\mathcal{V}})$ такие, что $\varkappa\left(T_{\delta}\right)<\delta, f(t) \in K_{\delta}$ и $\mathscr{F}(\cdot, t) \in \mathscr{K}_{\delta}$ при всех $t \in T_{\delta}$. Так как число $\delta>0$ можно выбрать сколь угодно мальм, а функции из $\mathscr{K}_{\delta}$ равностепенно непрерывны на компакте $K_{\delta}$, то отсюда следует, что для любого $\varepsilon>0$ можно найти такое число $\varepsilon^{\prime}>0$, что любой обший $\varepsilon^{\prime}$-почти период функций $f \in S_{1}\left(\mathbb{R},\left(\mathscr{U}, \rho^{\prime}\right)\right)$ и $\mathscr{F}(\cdot, \cdot)$ является также и $\varepsilon$-почти периодом измеримой функции $\mathscr{F}(f(\cdot), \cdot) \in\left(M(\mathbb{R}, \widetilde{\mathcal{V}}), D^{(\tilde{\rho})}\right)$. Общие $\varepsilon^{\prime}$-почти периоды функций $f$ и $\mathscr{F}(\cdot, \cdot)$ образуют относительно плотное множество [10], поэтому $\mathscr{F}(f(\cdot), \cdot) \in S(\mathbb{R}, \mathscr{V})$. Включение $\operatorname{Mod} \mathscr{F}(f(\cdot), \cdot) \subset \operatorname{Mod} f+\operatorname{Mod} \mathscr{F}(\cdot, \cdot)$ следует из того, что всякая последовательность $\left\{\tau_{j}\right\} \subset \mathbb{R}$, которая одновременно $f$ - и $\mathscr{F}(\cdot, \cdot)$-возврашаюшая, является также и $\mathscr{F}(f(\cdot), \cdot)$-возврашаюшей.

СлЕДСТВИЕ. Пусть $(\mathscr{U}, \rho)$ и $(\mathscr{V}, \tilde{\rho})$ - метрические пространства, при этом пространство $\mathscr{U}$ полное. Если $f \in S(\mathbb{R}, \mathscr{U})$ и $\mathscr{F} \in C(\mathscr{U}, \mathscr{V})$, то $\mathscr{F}(f(\cdot)) \in$ $S(\mathbb{R}, \mathscr{V}) u \operatorname{Mod} \mathscr{F}(f(\cdot)) \subset \operatorname{Mod} f$.

Для доказательства достаточно заметить, что функция $\mathscr{F}:(\mathscr{U}, \rho) \rightarrow\left(\mathscr{V}, \tilde{\rho}^{\prime}\right)$ непрерывна и ограничена.

Пусть $\mathscr{B}(\mathscr{U})$ - совокупность борелевских подмножеств полного метрического пространства $(\mathscr{U}, \rho), \mathscr{M}=\mathscr{M}(\mathscr{U})$ - множество борелевских вероятностных мер на пространстве $(\mathscr{U}, \rho)$. Каждой мере $\mu[\cdot] \in \mathscr{M}$ ставится в соответствие функционал

$$
\mu(\mathscr{F})=\int_{\mathscr{U}} \mathscr{F}(x) \mu[d x], \quad \mathscr{F} \in C_{b}(\mathscr{U}) .
$$

Для мер $\mu[\cdot] \in \mathscr{M}$ и соответствуюших им функционалов $\mu(\cdot)$ будет в дальнейшем использоваться одинаковое обозначение, но с разными скобками для аргумента. 
Пусть $\mu \in \mathscr{M}$. Множество точек $x \in \mathscr{U}$, для которых $\mu[K(x, \varepsilon)]>0$ при всех $\varepsilon>0$, образует носитель $\operatorname{supp} \mu$ меры $\mu, \operatorname{supp} \mu$ - замкнутое сепарабельное множество. Мера $\mu \in \mathscr{M}$ является вероятностей мерой Радона, если для любого $\varepsilon>0$ сушествует компакт $K_{\varepsilon} \subset \mathscr{U}$ такой, что $\mu\left[K_{\varepsilon}\right]>1-\varepsilon$. Множество вероятностных мер Радона обозначим через $\mathscr{M}_{\tau}=\mathscr{M}_{\tau}(\mathscr{U})$. Справедливо равенство $\mathscr{M}_{\tau}=\{\mu \in \mathscr{M}: \mu[\operatorname{supp} \mu]=1\}$. Если $\mathscr{U}$ - сепарабельное пространство, то $\mathscr{M}=\mathscr{M}_{\tau}$.

На множестве $\mathscr{M}$ вводится метрика Прохорова

$$
d(\mu, \nu)=\inf \left\{\varepsilon>0: \mu[A] \leqslant \nu\left[A^{\varepsilon}\right]+\varepsilon \text { для всех } A \in \mathscr{B}(\mathscr{U})\right\}, \quad \mu, \nu \in \mathscr{M},
$$

где $A^{\varepsilon}=\{x \in \mathscr{U}: \rho(x, \mathscr{A})<\varepsilon\}$. Метрическое пространство $\left(\mathscr{M}_{\tau}, d\right)$ является полным (вместе с пространством $(\mathscr{U}, \rho))$. Для любой функции $\mathscr{F} \in C_{b}(\mathscr{U})$ функция $\mathscr{M}_{\tau} \ni \mu \mapsto \mu(\mathscr{F}) \in \mathbb{R}$ непрерывна. Множество $\mathscr{K}$ предкомпактно в метрическом пространстве $\left(\mathscr{M}_{\tau}, d\right)$ тогда и только тогда, когда для любого $\varepsilon>0$ существует компакт $K_{\varepsilon} \subset \mathscr{U}$ такой, что $\mu\left[K_{\varepsilon}\right]>1-\varepsilon$ для всех мер $\mu \in \mathscr{K}$.

Пусть $\delta_{x}$ - мера Дирака, сосредоточенная в точке $x \in \mathscr{U}, \mathscr{M}_{\delta}$ - множество мер $\mu \in \mathscr{M}$, имеющих вид $\mu=\sum_{j=1}^{N} \lambda_{j} \delta_{x_{j}}$, где $N \in \mathbb{N}, \lambda_{j} \geqslant 0, \sum_{j=1}^{N} \lambda_{j}=1$ и $x_{j} \in \mathscr{U}$, $j=1, \ldots, N$. Множество $\mathscr{M}_{\delta}$ плотно в $\mathscr{M}_{\tau}$. Более того, для любой меры $\mu \in \mathscr{M}_{\tau}$ и любого $\varepsilon>0$ сушествует мера $\nu \in \mathscr{M}_{\delta}$, для которой $d(\mu, \nu)<\varepsilon$ и $\operatorname{supp} \nu \subset \operatorname{supp} \mu$.

Если $\mu_{j} \in \mathscr{M}_{\tau}, \lambda_{j} \geqslant 0, j \in \mathbb{N}$, и $\sum_{j=1}^{\infty} \lambda_{j}=1$, то $\mu=\sum_{j=1}^{\infty} \lambda_{j} \mu_{j} \in \mathscr{M}_{\tau}$.

Пусть $\mu \in \mathscr{M}_{\tau}, \mathscr{F} \in C_{b}^{+}(\mathscr{U})$ и $\mu(\mathscr{F})>0$. Обозначим через $\mu^{\mathscr{F}}[\cdot]$ вероятностную меру Радона, которой соответствует функционал

$$
f \mapsto \frac{1}{\mu(\mathscr{F})} \mu(\mathscr{F} f), \quad f \in C_{b}(\mathscr{U}) .
$$

Имеем $\operatorname{supp} \mu^{\mathscr{F}} \subset \operatorname{supp} \mu \cap \operatorname{supp} \mathscr{F}$, где

$$
\operatorname{supp} \mathscr{F}=\overline{\{x \in \mathscr{U}: \mathscr{F}(x) \neq 0\}} .
$$

На множестве $\mathscr{M}_{\tau} \times C_{\boldsymbol{b}}(\mathscr{U})$ введем метрику

$$
D^{0}\left(\left(\mu_{1}, \mathscr{F}_{1}\right),\left(\mu_{2}, \mathscr{F}_{2}\right)\right)=d\left(\mu_{1}, \mu_{2}\right)+\left\|\mathscr{F}_{1}-\mathscr{F}_{2}\right\|,
$$

где $\mu_{1}, \mu_{2} \in \mathscr{M}_{\tau}, \mathscr{F}_{1}, \mathscr{F}_{2} \in C_{b}(\mathscr{U})$. Пространство $\left(\mathscr{M}_{\tau} \times C_{b}(\mathscr{U}), D^{0}\right)$ полное. Отображение $\mathscr{M}_{\tau} \times C_{b}(\mathscr{U}) \ni(\mu, \mathscr{F}) \mapsto \mu^{\mathscr{F}} \in \mathscr{M}_{\tau}$ непрерьвно на своей области определения $\left\{(\mu, \mathscr{F}) \in \mathscr{M}_{\tau} \times C_{b}(\mathscr{U}): \mathscr{F} \in C_{b}^{+}(\mathscr{U}), \mu(\mathscr{F})>0\right\}$.

Мерозначная функция $t \mapsto \mu[\cdot ; t] \in \mathscr{M}_{\tau}, t \in \mathbb{R}$, измерима, если функция $\mu[\cdot ; \cdot] \in M\left(\mathbb{R},\left(\mathscr{M}_{\tau}, d\right)\right)$. Из определения измеримости следует, что для любой функции $\mu[\cdot ; \cdot] \in M\left(\mathbb{R}, \mathscr{M}_{\tau}\right)$ сушествует замкнутое сепарабельное множество $Q=Q(\mu[\cdot ; \cdot]) \subset \mathscr{U}$ такое, что $\operatorname{supp} \mu[\cdot ; t] \subset Q$ п.в.

Мерозначная функция $\mu[\cdot ; \cdot] \in M\left(\mathbb{R}, \mathscr{M}_{\tau}\right)$ называется почти периодической по Степанову, если

$$
\mu[\cdot ; \cdot] \in S\left(\mathbb{R},\left(\mathscr{M}_{\tau}, d\right)\right)=S_{1}\left(\mathbb{R},\left(\mathscr{M}_{\tau}, d\right)\right) .
$$

Для любых $x, y \in \mathscr{U}$ справедливо равенство $d\left(\delta_{x}, \delta_{y}\right)=\rho^{\prime}(x, y)$, поэтому функция $f: \mathbb{R} \rightarrow \mathscr{U}$ принадлежит пространству $S(\mathbb{R}, \mathscr{U})$ тогда и только тогда, когда $\delta_{f(\cdot)} \in S\left(\mathbb{R}, \mathscr{M}_{\tau}\right)$, при этом $\operatorname{Mod} f=\operatorname{Mod} \delta_{f(\cdot)}$. 
Лemma 4. Пусть $\mu_{j}[\cdot ; \cdot] \in S\left(\mathbb{R}, \mathscr{M}_{\tau}\right), \lambda_{j}(\cdot) \in S(\mathbb{R}, \mathbb{R}), j \in \mathbb{N}, \lambda_{j}(t) \geqslant 0 u$ $\sum_{j} \lambda_{j}(t)=1$ n.e.,

$$
\lim _{N \rightarrow \infty} \sup _{\xi \in \mathbb{R}} \sum_{j \geqslant N} \int_{\xi}^{\xi+1} \lambda_{j}(t) d t=0 .
$$

Тогда $\mu[\cdot ; \cdot] \doteq \sum_{j} \lambda_{j}(\cdot) \mu_{j}[\cdot ; \cdot] \in S\left(\mathbb{R}, \mathscr{M}_{\tau}\right) \quad u \quad \operatorname{Mod} \mu[\cdot ; \cdot] \subset \sum_{j} \operatorname{Mod} \lambda_{j}+$ $\sum_{j} \operatorname{Mod} \mu_{j}[\cdot ; \cdot] . \quad$ Eсли, кроме того, $\mu_{j}[\cdot ; \cdot] \in S^{c}\left(\mathbb{R}, \mathscr{M}_{\tau}\right), j=1, \ldots, N, u$ $\lambda_{j}(t)=0$ n.в. при всех $j>N$, где $N \in \mathbb{N}$, то $\mu[\cdot ; \cdot] \in S^{c}\left(\mathbb{R}, \mathscr{M}_{\tau}\right)$.

ДоказАтельство. Для всех $N \in \mathbb{N}$ обозначим через $L_{N}$ множество наборов $\left\{\lambda_{j}^{\prime}, \mu_{j}^{\prime}\right\}_{j=1, \ldots, N}$ чисел $\lambda_{j}^{\prime} \geqslant 0$, для которых $\sum_{j=1}^{N} \lambda_{j}^{\prime} \leqslant 1$, и мер $\mu_{j}^{\prime} \in \mathscr{M}_{\tau}$. Множество $L_{N}$ становится полным метрическим пространством, если ввести метрику

$$
\mathscr{R}_{N}\left(\left\{\lambda_{j}^{\prime}, \mu_{j}^{\prime}\right\},\left\{\lambda_{j}^{\prime \prime}, \mu_{j}^{\prime \prime}\right\}\right)=\sum_{j=1}^{N}\left|\lambda_{j}^{\prime}-\lambda_{j}^{\prime \prime}\right|+\sum_{j=1}^{N} d\left(\mu_{j}^{\prime}, \mu_{j}^{\prime \prime}\right)
$$

Функция $t \mapsto\left\{\lambda_{j}(t), \mu_{j}[\cdot ; t]\right\}_{j=1, \ldots, N} \in\left(L_{N}, \mathscr{R}_{N}\right), t \in \mathbb{R}$, почти периодична по Степанову и ее модуль совпадает с суммой модулей функций $\lambda_{j}$ и $\mu_{j}[\cdot ; \cdot]$, $j=1, \ldots, N$. Так как отображение $\mathscr{B}_{N}: L_{N} \rightarrow \mathscr{M}_{\tau}$, ставящее в соответствие каждому набору $\left\{\lambda_{j}^{\prime}, \mu_{j}^{\prime}\right\} \in\left(L_{N}, \mathscr{R}_{N}\right)$ меру

$$
\sum_{j=1}^{N} \lambda_{j}^{\prime} \mu_{j}^{\prime}+\left(1-\sum_{j=1}^{N} \lambda_{j}^{\prime}\right) \delta_{x_{0}} \in\left(\mathscr{M}_{\tau}, d\right)
$$

непрерывно, то в силу следствия леммы 3 имеем

$$
\begin{gathered}
\mu^{N}[\cdot ; \cdot] \doteq \sum_{j=1}^{N} \lambda_{j}(\cdot) \mu_{j}[\cdot ; \cdot]+\left(1-\sum_{j=1}^{N} \lambda_{j}(\cdot)\right) \delta_{x_{0}}[\cdot] \in S\left(\mathbb{R}, \mathscr{M}_{\tau}\right), \\
\operatorname{Mod} \mu^{N}[\cdot ; \cdot] \subset \sum_{j=1}^{N} \operatorname{Mod} \lambda_{j}+\sum_{j=1}^{N} \operatorname{Mod} \mu_{j}[\cdot ; \cdot] .
\end{gathered}
$$

Из $(2)$ следует, что $\mu[\cdot ; \cdot] \in M\left(\mathbb{R}, \mathscr{M}_{\tau}\right)$ и $D^{(d)}\left(\mu, \mu^{N}\right) \rightarrow 0$ при $N \rightarrow \infty$, поэтому $\mu[\cdot ; \cdot] \in S\left(\mathbb{R}, \mathscr{M}_{\tau}\right)$ и выполняется требуемое включение для $\operatorname{Mod} \mu[\cdot ; \cdot]$. Пусть теперь $\mu_{j}[\cdot ; \cdot] \in S^{c}\left(\mathbb{R}, \mathscr{M}_{\tau}\right), j=1, \ldots, N, \lambda_{j}(t)=0$ п.в. при всех $j>N$, где $N \in \mathbb{N}$. Найдутся компакты $\mathscr{K}_{j} \subset \mathscr{M}_{\tau}, j=1, \ldots, N$, такие, что $\mu_{j}[\cdot ; t] \in \mathscr{K}_{j}$ п.в. Тогда множество $\mathscr{K}=\left\{\sum_{j=1}^{N} \lambda_{j} \mu_{j}: \lambda_{j} \geqslant 0, \sum_{j=1}^{N} \lambda_{j}=1\right.$ и $\left.\mu_{j} \in \mathscr{K}_{j}\right\}$ также компактное и $\mu[\cdot ; t] \in \mathscr{K}$ п.в. Лемма 4 доказана.

Пусть cl $\mathscr{U}$ - множество непустых замкнутых ограниченных подмножеств метрического пространства $(\mathscr{U}, \rho)$. На множестве сl $\mathscr{U}$ вводится метрика Хаусдорфа $\operatorname{dist}(\cdot, \cdot)=\operatorname{dist}_{\rho}(\cdot, \cdot)$. Так как пространство $\mathscr{U}$ предполагается полным, то (cl $\mathscr{U}$, dist) - также полное метрическое пространство. На множестве $\mathrm{cl}^{\prime} \mathscr{U} \doteq \mathrm{cl}\left(\mathscr{U}, \rho^{\prime}\right)$ непустых замкнутых подмножеств метрического пространства 
$(\mathscr{U}, \rho)$ вводится метрика Хаусдорфа dist $\rho^{\prime}(\cdot, \cdot)$ (соответствуюшая метрике $\left.\rho^{\prime}\right)$. Для множеств $A, B \in \mathrm{cl} \mathscr{U}$ имеем

$$
\operatorname{dist}^{\prime}(A, B) \doteq \theta\left(\operatorname{dist}_{\rho}(A, B)\right)=\operatorname{dist}_{\rho^{\prime}}(A, B),
$$

поэтому $\left(\mathrm{cl} \mathscr{U}\right.$, dist $\left.^{\prime}\right)$ - замкнутое подпространство полного метрического пространства $\left(\mathrm{cl}^{\prime} \mathscr{U}, \operatorname{dist}_{\rho^{\prime}}\right)$. Для множества $A \in \mathrm{cl}^{\prime} \mathscr{U}$ обозначим

$$
\operatorname{diam} A=\sup \{\rho(x, y): x, y \in A\} .
$$

\section{§2. Равномерная аппроксимация почти периодических функций}

Теорема 1. Для любой функции $f \in S(\mathbb{R}, \mathscr{U})$ и любого $\varepsilon>0$ найдутся последовательность $\left\{T_{j}\right\} \in \mathfrak{M}(\operatorname{Mod} f)$ и точки $x_{j} \in \mathscr{U}, j \in \mathbb{N}$, такие, что функиия $f(t)$ определена при всех $t \in \bigcup T_{j}$, множества $\left\{f(t): t \in T_{j}\right\}$ предкомпактны для всех $j \in \mathbb{N} u$

$$
\sup _{t \in \cup T_{j}} \rho\left(f(t), \sum x_{j} \chi_{T_{j}}(t)\right)<\varepsilon
$$

( из леммы 2 следует, ито $\left.\sum x_{j} \chi_{T_{j}}(\cdot) \in S(\mathbb{R}, \mathscr{U}) u \operatorname{Mod} \sum x_{j} \chi_{T_{j}}(\cdot) \subset \operatorname{Mod} f\right)$.

Теорема 1 является следствием следуюшей теоремы, доказательство которой приведено в [2], [6].

Tеорема 2. Пусть $f \in S(\mathbb{R}, \mathscr{U})$. Тогда для любого $\varepsilon>0$ найдутся последовательность $\left\{T_{j}\right\} \in \mathfrak{M}(\operatorname{Mod} f)$ и точки $x_{j} \in \mathscr{U}, j \in \mathbb{N}$, такие, что

$$
D_{\infty}^{(\rho)}\left(f(\cdot), \sum x_{j} \chi_{T_{j}}(\cdot)\right)<\varepsilon
$$

ДоКАЗАТЕЛЬСТВо ТЕОРЕМЫ 1 . Из теоремы 2 следует, что для любого $i \in \mathbb{N}$ сушествуют последовательность $\left\{T_{n}^{(i)}\right\} \in \mathfrak{M}(\operatorname{Mod} f)$ и точки $x_{n}^{(i)} \in \mathscr{U}, n \in \mathbb{N}$, такие, что функция $f(t)$ определена при всех $t \in \bigcup_{n} T_{n}^{(i)}$ и

$$
\sup _{t \in \cup_{n} T_{n}^{(i)}} \rho\left(f(t), \sum_{n} x_{n}^{(i)} \chi_{T_{n}^{(i)}}(t)\right)<2^{-i} .
$$

Для любых $i, m \in \mathbb{N}$ можно найти такое число $N(i, m) \in \mathbb{N}$, что

$$
\varkappa\left(\bigcup_{n \leqslant N(i, m)} T_{n}^{(i)}\right)<2^{-i-m} .
$$

Обозначим

$$
T_{m}^{\prime}=\bigcap_{i} \bigcup_{n \leqslant N(i, m)} T_{n}^{(i)}, \quad m \in \mathbb{N} .
$$

Имеем $T_{m}^{\prime} \in S(\mathbb{R}), \operatorname{Mod} T_{m}^{\prime} \subset \operatorname{Mod} f$ и $\varkappa\left(T_{m}^{\prime}\right)<2^{-m}$. Для всех $i \in \mathbb{N}$ множества $\left\{x_{n}^{(i)}: n=1, \ldots, N(i, m)\right\}$ образуют конечную $2^{-i}$-сеть для множеств 
$\left\{f(t): t \in T_{m}^{\prime}\right\}$, поэтому последние множества предкомпактны. Положим $T_{1}^{\prime \prime}=T_{1}^{\prime}$ и $T_{m}^{\prime \prime}=T_{m}^{\prime} \backslash \bigcup_{l<m} T_{l}^{\prime}$ при $m \geqslant 2$. Тогда $T_{m}^{\prime \prime} \in S(\mathbb{R}), \operatorname{Mod} T_{m}^{\prime \prime} \subset \operatorname{Mod} f$ и $\varkappa\left(\bigcup_{l \leqslant m} T_{l}^{\prime \prime}\right)<2^{-m}$ для всех $m \in \mathbb{N}$. Отсюда $\left\{T_{m}^{\prime \prime}\right\} \in \mathfrak{M}(\operatorname{Mod} f)$. При этом функция $f(t)$ определена при всех $t \in \bigcup T_{m}^{\prime \prime}$ и $\left\{f(t): t \in T_{m}^{\prime \prime}\right\}$ - предкомпактные множества для всех $m \in \mathbb{N}$. Выберем индекс $a \in \mathbb{N}$ так, что $2^{-a}<\varepsilon$. Пусть $T_{j}$, $j \in \mathbb{N},-$ как-то перенумерованные множества $T_{m}^{\prime \prime} \cap T_{n}^{(a)}, m, n \in \mathbb{N}$. Каждому индексу $j$ ставится в соответствие индекс $n=n(j)$ (и индекс $m=m(j)$ ). Положим $x_{j}=x_{n(j)}^{(a)}$. Требуемые последовательность $\left\{T_{j}\right\} \in \mathfrak{M}(\operatorname{Mod} f)$ и точки $x_{j}, j \in \mathbb{N}$, найдены. Теорема доказана.

Теорема 2 позволяет дать другое (эквивалентное введенному) определение пространства $S(\mathbb{R}, \mathscr{U}):$ функция $f \in M(\mathbb{R}, \mathscr{U})$ принадлежит пространству $S(\mathbb{R}, \mathscr{U})$ тогда и только тогда, когда для любого $\varepsilon>0$ существуют последовательность $\left\{T_{j}\right\} \in \mathfrak{M}(\mathbb{R})$ и точки $x_{j} \in \mathscr{U}, j \in \mathbb{N}$, такие, что вьполняется неравенство (3).

\section{§3. Основные результаты}

Многозначное отображение $t \mapsto F(t) \in \mathrm{cl}^{\prime} \mathscr{U}, t \in \mathbb{R}$, для которого $F(t)=$ $\overline{\bigcup_{j} f_{j}(t)}$ п.в., где $f_{j} \in S(\mathbb{R}, \mathscr{U}), j \in \mathbb{N}$, представимо в виде $F(t)=\operatorname{supp} \mu[\cdot ; t]$ п.в., $\mu[\cdot ; t] \doteq \sum_{j} 2^{-j} \delta_{f_{j}(t)}[\cdot], t \in \mathbb{R}$. При этом (в силу леммы 4$) \mu[\cdot ; \cdot] \in S\left(\mathbb{R}, \mathscr{M}_{\tau}\right)$ и $\operatorname{Mod} \mu[\cdot ; \cdot] \subset \sum_{j} \operatorname{Mod} \delta_{f_{j}(\cdot)}=\sum_{j} \operatorname{Mod} f_{j}$. Если $f_{j} \in S^{c}(\mathbb{R}, \mathscr{U}), j \in \mathbb{N}$, то $\delta_{f_{j}(\cdot)} \in S^{c}\left(\mathbb{R}, \mathscr{M}_{\tau}\right)$ и из леммы 4 получаем

$$
2^{-N} \delta_{x_{0}}+\sum_{j=1}^{N} 2^{-j} \delta_{f_{j}(\cdot)} \in S^{c}\left(\mathbb{R}, \mathscr{M}_{\tau}\right)
$$

для всех $N \in \mathbb{N}$. Так как

$$
d\left(\mu[\cdot ; t], 2^{-N} \delta_{x_{0}}[\cdot]+\sum_{j=1}^{N} 2^{-j} \delta_{f_{j}(t)}[\cdot]\right) \leqslant 2^{-N}
$$

для всех $N \in \mathbb{N}$ и п.в. $t \in \mathbb{R}$, то $\mu[\cdot ; \cdot] \in S^{c}\left(\mathbb{R}, \mathscr{M}_{\tau}\right)$.

ТеОрема 3. Пусть $(\mathscr{U}, \rho)$ - полное метрическое пространство, $\mu[\cdot ; \cdot] \in$ $S^{c}\left(\mathbb{R}, \mathscr{M}_{\tau}\right)$. Тогда существует счетное множество функиий $f_{j} \in S^{c}(\mathbb{R}, \mathscr{U})$, $j \in \mathbb{N}$, maкux, что $\operatorname{Mod} f_{j} \subset \operatorname{Mod} \mu[\cdot ; \cdot] u \operatorname{supp} \mu[\cdot ; t]=\overline{\bigcup_{j} f_{j}(t)}$ n.в.

При доказательстве теоремы 3 используется теорема 4 , доказательство которой приведено в $\S 4$.

Для всех $\mu \in \mathscr{M}_{\tau}, x \in \mathscr{U}$ и $\varepsilon \in(0,1)$ обозначим

$$
r_{\varepsilon}(\mu, x)=\min \{r \geqslant 0: \mu[K(x, r)] \geqslant \varepsilon\} .
$$

TЕОРема 4. Пусть $(\mathscr{U}, \rho)$ - полное метрическое пространство, $\mu[\cdot ; \cdot] \in$ $S^{c}\left(\mathbb{R}, \mathscr{M}_{\tau}\right)$ и $g \in S^{c}(\mathbb{R}, \mathscr{U})$. Тогда для любого $\varepsilon \in(0,1)$ существует функиия $f \in S^{c}(\mathbb{R}, \mathscr{U})$ maкая, umo $\operatorname{Mod} f \subset \operatorname{Mod} \mu[\cdot ; \cdot]+\operatorname{Mod} g, f(t) \in \operatorname{supp} \mu[\cdot ; t]$ n.в. u $\rho(f(t), g(t))<r_{\varepsilon}(\mu[\cdot ; t], g(t))+\varepsilon$ n.в. 
ДОКАЗАТЕЛЬСТво ТЕОРЕМЫ 3 . Пусть $Q=Q(\mu[\cdot ; \cdot]) \subset \mathscr{U}$ - сепарабельное множество такое, что $\operatorname{supp} \mu[\cdot ; t] \subset Q$ п.в., и точки $x_{j} \in Q, j \in \mathbb{N}$, образуют счетное плотное в $Q$ множество. В силу теоремы 4 найдутся множество $T \subset \mathbb{R}$, $\operatorname{mes}(\mathbb{R} \backslash T)=0$, и функции $f_{i j} \in S^{c}(\mathbb{R}, \mathscr{U}), i, j \in \mathbb{N}$, такие, что функции $\mu[\cdot ; t]$ и $f_{i j}(t)$, $i, j \in \mathbb{N}$, определены при всех $t \in T, \operatorname{Mod} f_{i j} \subset \operatorname{Mod} \mu[\cdot ; \cdot], f_{i j}(t) \in \operatorname{supp} \mu[\cdot ; t] \subset Q$ (отсюда $\left.\overline{\bigcup_{i, j} f_{i j}(t)} \subset \operatorname{supp} \mu[\cdot ; t]\right)$ и $\rho\left(f_{i j}(t), x_{j}\right)<r_{1 /(i+1)}\left(\mu[\cdot ; t], x_{j}\right)+1 /(i+1)$ при всех $t \in T$. Пусть $t \in T$ и $x \in \operatorname{supp} \mu[\cdot ; t]$. Для любого $\varepsilon>0$ найдется точка $x_{j} \in Q$ такая, что $\rho\left(x, x_{j}\right)<\varepsilon / 4$. Так как $x \in \operatorname{supp} \mu[\cdot ; t]$, то $\mu\left[K\left(x_{j}, \varepsilon / 2\right) ; t\right]>0$. Если $1 /(i+1)<\min \left\{\varepsilon / 4, \mu\left[K\left(x_{j}, \varepsilon / 2\right) ; t\right]\right\}$, то $r_{1 /(i+1)}\left(\mu[\cdot ; t], x_{j}\right) \leqslant \varepsilon / 2$ и, следовательно, $\rho\left(f_{i j}(t), x\right) \leqslant \rho\left(x, x_{j}\right)+\rho\left(f_{i j}(t), x_{j}\right)<\varepsilon / 4+\varepsilon / 2+\varepsilon / 4=\varepsilon$. Число $\varepsilon>0$ можно выбрать произвольно, поэтому $x \in \overline{\bigcup_{i, j} f_{i j}(t)}$. Из произвольности выбора числа $t \in T$ и точки $x \in \operatorname{supp} \mu[\cdot ; t]$ получим $\operatorname{supp} \mu[\cdot ; t]=\overline{\bigcup_{i, j} f_{i j}(t)}$ при всех $t \in T$. Осталось перенумеровать функции $f_{i j}$ с помощью одного индекса. Теорема доказана.

Другое доказательство теоремы 3 в случае компактного метрического пространства $(\mathscr{U}, \rho)$ приведено в $[8]$.

Следствием теорем 3 и 4 являются теоремы $3^{\prime}$ и $4^{\prime}$.

Tеорема $3^{\prime}$. Пусть $(\mathscr{U}, \rho)$ - полное метрическое пространство, $\mu[\cdot ; \cdot] \in$ $S\left(\mathbb{R}, \mathscr{M}_{\tau}\right)$. Тогда существует счетное множество функиий $f_{j} \in S(\mathbb{R}, \mathscr{U})$, $j \in \mathbb{N}$, maкux, ито $\operatorname{Mod} f_{j} \subset \operatorname{Mod} \mu[\cdot ; \cdot] u \operatorname{supp} \mu[\cdot ; t]=\overline{\bigcup_{j} f_{j}(t)}$ n.в.

ДокАЗАТЕльство. В силу теоремы 1 найдется последовательность множеств $\left\{T_{i}\right\} \in \mathfrak{M}(\operatorname{Mod} \mu[\cdot ; \cdot])$ такая, что мерозначная функция $\mu[\cdot ; t]$ определена при всех $t \in \bigcup_{i} T_{i}$ и $\bigcup_{t \in T_{i}} \mu[\cdot ; t] \subset \mathscr{M}_{\tau}$ - предкомпактные множества, $i \in \mathbb{N}$. Определим мерозначные функции

$$
\mu_{i}[\cdot ; t]= \begin{cases}\mu[\cdot ; t], & \text { если } t \in T_{i}, \\ \delta_{x_{0}}[\cdot], & \text { если } t \notin T_{i} .\end{cases}
$$

Из леммы 2 следует $\mu_{i}[\cdot ; \cdot] \in S^{c}\left(\mathbb{R}, \mathscr{M}_{\tau}\right)$ и $\operatorname{Mod} \mu_{i}[\cdot ; \cdot] \subset \operatorname{Mod} \mu[\cdot ; \cdot], i \in \mathbb{N}$. Для каждого $i \in \mathbb{N}$ (в силу теоремы 3 ) найдутся функции $f_{i j} \in S(\mathbb{R}, \mathscr{U}), j \in \mathbb{N}$, такие, что $\operatorname{Mod} f_{i j} \subset \operatorname{Mod} \mu_{i}[\cdot ; \cdot]$ и $\overline{\bigcup_{j} f_{i j}(t)}=\operatorname{supp} \mu_{i}[\cdot ; t]$ п.в. Положим

$$
f_{j}(\cdot)=\sum_{i} f_{i j}(\cdot) \chi_{T_{i}}(\cdot)
$$

Снова используя лемму 2 , получаем $f_{j} \in S(\mathbb{R}, \mathscr{U}), \operatorname{Mod} f_{j} \subset \sum_{i} \operatorname{Mod} f_{i j}+$ $\sum_{i} \operatorname{Mod} T_{i} \subset \operatorname{Mod} \mu[\cdot ; \cdot], j \in \mathbb{N}$. При этом $\overline{\bigcup_{j} f_{j}(t)}=\operatorname{supp} \mu[\cdot ; t]$ п.в. Теорема $3^{\prime}$ доказана.

ТеОРема $4^{\prime}$. Пусть $(\mathscr{U}, \rho)$ - полное метрическое пространство, $\mu[\cdot ; \cdot] \in$ $S\left(\mathbb{R}, \mathscr{M}_{\tau}\right)$ и $g \in S(\mathbb{R}, \mathscr{U})$. Тогда для любого $\varepsilon \in(0,1)$ существует функиия $f \in S(\mathbb{R}, \mathscr{U})$ такая, ито $\operatorname{Mod} f \subset \operatorname{Mod} \mu[\cdot ; \cdot]+\operatorname{Mod} g, f(t) \in \operatorname{supp} \mu[\cdot ; t]$ n.в. $u$ $\rho(f(t), g(t))<r_{\varepsilon}(\mu[\cdot ; t], g(t))+\varepsilon$ n.в.

Теорема $4^{\prime}$ доказывается аналогично теореме $3^{\prime}$ с использованием леммы 2 и теорем 1,4 . 
TеОрема 5. Пусть $(\mathscr{U}, \rho)$ - полное метрическое пространство, $\mu[\cdot ; \cdot] \in$ $S\left(\mathbb{R}, \mathscr{M}_{\tau}\right)$. Если существует функиия $f \in S^{c}(\mathbb{R}, \mathscr{U}) \quad\left(f \in S(\mathbb{R}, \mathscr{U}) \cap L^{\infty}(\mathbb{R}, \mathscr{U})\right.$ или $\left.f \in S_{p}(\mathbb{R}, \mathscr{U}), p \geqslant 1\right)$ такая, что $f(t) \in \operatorname{supp} \mu[\cdot ; t]$ n.в., то найдется счетное множество функиий $\tilde{f}_{j} \in S^{c}(\mathbb{R}, \mathscr{U}) \quad\left(\tilde{f}_{j} \in S(\mathbb{R}, \mathscr{U}) \cap L^{\infty}(\mathbb{R}, \mathscr{U})\right.$ или $\left.\underline{\tilde{f}_{j} \in S_{p}}(\mathbb{R}, \mathscr{U})\right), j \in \mathbb{N}$, maкux, ито $\operatorname{Mod} \tilde{f}_{j} \subset \operatorname{Mod} \mu[\cdot ; \cdot]+\operatorname{Mod} f u \operatorname{supp} \mu[\cdot ; t]=$ $\overline{\bigcup_{j} \tilde{f}_{j}(t)}$ n.в.

ДоказАТЕЛЬство. Пусть $f_{j}, j \in \mathbb{N},-$ функции, сушествование которых доказывается в теореме $3^{\prime}$. В силу теоремы 1 для любых $i, j \in \mathbb{N}$ можно найти множества $T_{j}^{(i)} \in S(\mathbb{R})$ такие, что $\operatorname{Mod} T_{j}^{(i)} \subset \operatorname{Mod} f_{j}, \varkappa\left(T_{j}^{(i)}\right)<1 / i$, функции $f_{j}(t)$ определены при всех $t \in \bigcup_{i} T_{j}^{(i)}$ и $\left\{f_{j}(t): t \in T_{j}^{(i)}\right\}$ - предкомпактные (следовательно, ограниченные) множества в метрическом пространстве $(\mathscr{U}, \rho)$. Определим функции

$$
\tilde{f}_{i j}(t)=\left\{\begin{array}{l}
f_{j}(t), \text { если } t \in T_{j}^{(i)}, \\
f(t), \text { если } t \notin T_{j}^{(i)} .
\end{array}\right.
$$

Из леммы 2 следует, что $\tilde{f}_{i j} \in S^{c}(\mathbb{R}, \mathscr{U})$ (если $f \in S(\mathbb{R}, \mathscr{U}) \cap L^{\infty}(\mathbb{R}, \mathscr{U})$, то $\tilde{f}_{i j} \in$ $S(\mathbb{R}, \mathscr{U}) \cap L^{\infty}(\mathbb{R}, \mathscr{U})$, если $f \in S_{p}(\mathbb{R}, \mathscr{U})$, то также $\left.\tilde{f}_{i j} \in S_{p}(\mathbb{R}, \mathscr{U})\right), i, j \in \mathbb{N}$, и $\operatorname{Mod} \tilde{f}_{i j} \subset \operatorname{Mod} f_{j}+\operatorname{Mod} f \subset \operatorname{Mod} \mu[\cdot ; \cdot]+\operatorname{Mod} f$. Так как $\operatorname{mes}\left(\mathbb{R} \backslash \bigcap_{j} \bigcup_{i} T_{j}^{(i)}\right)=0$, то $\operatorname{supp} \mu[\cdot ; t]=\overline{\bigcup_{i, j} \tilde{f}_{i j}(t)}$ п.в. Осталось перенумеровать функции $\tilde{f}_{i j}$ с помощью одного индекса. Теорема доказана.

ЗАмечаниЕ. Если $\mu[\cdot ; \cdot] \in S\left(\mathbb{R}, \mathscr{M}_{\tau}\right)$ и $r_{\varepsilon}\left(\mu[\cdot ; \cdot], x_{0}\right) \in M_{p}^{\prime}(\mathbb{R}, \mathbb{R})$ для некоторых $\varepsilon \in(0,1)$ и $p \geqslant 1\left(r_{\varepsilon}\left(\mu[\cdot ; \cdot], x_{0}\right) \in L^{\infty}(\mathbb{R}, \mathbb{R})\right.$ для некоторого $\left.\varepsilon \in(0,1)\right)$, то из (1) и теоремы $4^{\prime}$ вытекает существование функции $f \in S_{p}(\mathbb{R}, \mathscr{U})(f \in S(\mathbb{R}, \mathscr{U}) \cap$ $\left.L^{\infty}(\mathbb{R}, \mathscr{U})\right)$ такой, что $\operatorname{Mod} f \subset \operatorname{Mod} \mu[\cdot ; \cdot]$ и $f(t) \in \operatorname{supp} \mu[\cdot ; t]$ п.в.

В [3] на основе результатов работы [14] доказано, что в сепарабельном банаховом пространстве $(\mathscr{U},\|\cdot\|)$ любое многозначное отображение $F \in S_{1}(\mathbb{R},($ cl $\mathscr{U}$, dist $))$ можно представить в виде $F(t)=\overline{\bigcup_{j} f_{j}(t)}$ п.в., где $f_{j} \in S_{1}(\mathbb{R}, \mathscr{U})$ и $\operatorname{Mod} f_{j} \subset$ $\operatorname{Mod} F, j \in \mathbb{N}$. C помощью теоремы Фреше [15, с. 175$]$ отсюда нетрудно получить следуюшую теорему.

ТЕОРема 6. Пусть $(\mathscr{U}, \rho)$ - полное сепарабельное метрическое пространство и $F \in S\left(\mathbb{R},\left(\mathrm{cl}^{\prime} \mathscr{U}\right.\right.$, dist $\left.\left._{\rho^{\prime}}\right)\right)=S_{1}\left(\mathbb{R},\left(\mathrm{cl}^{\prime} \mathscr{U}, \operatorname{dist}_{\rho^{\prime}}\right)\right)$. Тогда существует счетное множество функиий $f_{j} \in S(\mathbb{R}, \mathscr{U}), j \in \mathbb{N}, \operatorname{maких,~ито~} \operatorname{Mod} f_{j} \subset \operatorname{Mod} F$ u $F(t)=\overline{\bigcup_{j} f_{j}(t)}$ n.в.

Другое доказательство теоремы 6, основанное на теореме 2, приведено в [2], [6] (при доказательстве нужно перейти к пространству $\left(\mathscr{U}, \rho^{\prime}\right)$ ).

ЗАмечание. Если в условиях теоремы $6 F \in M_{p}^{\prime}(\mathbb{R},(\mathrm{cl} \mathscr{U}$, dist)) (тогда $F \in$ $\left.S_{p}(\mathbb{R}, \mathrm{cl} \mathscr{U})\right), p \geqslant 1$, то все функции $f_{j}, j \in \mathbb{N}$, принадлежат пространству $S_{p}(\mathbb{R}, \mathscr{U})$. Действительно, в этом случае $\operatorname{dist}\left(F(\cdot),\left\{x_{0}\right\}\right) \in M_{p}^{\prime}(\mathbb{R}, \mathbb{R})$. Так как $\rho\left(f_{j}(t), x_{0}\right) \leqslant$ $\operatorname{dist}\left(F(t),\left\{x_{0}\right\}\right)$ п.в., то $f_{j} \in M_{p}^{\prime}(\mathbb{R}, \mathscr{U}), j \in \mathbb{N}$. Осталось воспользоваться равенством (1).

Если $\mu[\cdot ; \cdot] \in S\left(\mathbb{R}, \mathscr{M}_{\tau}\right)$, то не обязательно $\operatorname{supp} \mu[\cdot ; \cdot] \in S\left(\mathbb{R},\left(\mathrm{cl}^{\prime} \mathscr{U}, \operatorname{dist}_{\rho^{\prime}}\right)\right)$. Пример такой мерозначной функции $\mu[\cdot ; \cdot]$ (для компактного метрического пространства $\mathscr{U})$ приведен в [16]. 
Теорему 6 можно вывести также из теоремы $3^{\prime}$, показав, что любое многозначное отображение $F \in S\left(\mathbb{R},\left(\mathrm{cl}^{\prime} \mathscr{U}, \operatorname{dist}_{\rho^{\prime}}\right)\right)$ представимо в виде $F(t)=\operatorname{supp} \mu[\cdot ; t]$ п.в. для некоторой мерозначной функции $\mu[\cdot ; \cdot] \in S\left(\mathbb{R}, \mathscr{M}_{\tau}\right), \operatorname{Mod} \mu[\cdot ; \cdot] \subset \operatorname{Mod} F$. В случае компактного метрического пространства $\mathscr{U}$, но в более общей ситуации, когда требуется непрерывная зависимость многозначного отображения и его сечений от параметра, это сделано в [9].

ТЕОРема 7 [17]. Пусть $(\mathscr{U}, \rho)$ - полное метрическое пространство, $\mu[\cdot ; \cdot]$ $\in S^{c}\left(\mathbb{R}, \mathscr{M}_{\tau}\right) \quad\left(\mu[\cdot ; \cdot] \in S\left(\mathbb{R}, \mathscr{M}_{\tau}\right)\right)$. Тогда для любого $\varepsilon>0$ существует многозначное отображение $F(\cdot) \in S^{c}(\mathbb{R},(\mathrm{cl} \mathscr{U}, \mathrm{dist})) \quad(F(\cdot) \in S(\mathbb{R}, \mathrm{cl} \mathscr{U}))$ такое, что $F(t)$ - непустые компактные множества при п.в. $t \in \mathbb{R}$, $F(t) \subset \operatorname{supp} \mu[\cdot ; t]$ n.в., $\mu[F(t) ; t] \geqslant 1-\varepsilon$ n.в., при п.в. $t \in \mathbb{R}$ для любых $x \in F(t)$ $u \delta>0$ выполняется неравенство $\mu[F(t) \cap K(x, \delta) ; t]>0 u$

$$
\lim _{\Delta \rightarrow+0} \operatorname{ess} \sup \mu\left[F^{\Delta}(t) \backslash F(t) ; t\right]=0,
$$

əде $F^{\Delta}(t)=\{x \in \mathscr{U}: \rho(x, F(t))<\Delta\}, t \in \mathbb{R}$.

Tеорема 8. Пусть $(\mathscr{U}, \rho)$ - полное метрическое пространство, $\mu[\cdot ; \cdot] \in$ $S^{c}\left(\mathbb{R}, \mathscr{M}_{\tau}\right)$. Тогда для любого $\varepsilon>0$ найдутся иисло $N \in \mathbb{N}$ и функиии $f_{j} \in$ $S^{c}(\mathbb{R}, \mathscr{U}), j=1, \ldots, N$, mакие, ито $\operatorname{Mod} f_{j} \subset \operatorname{Mod} \mu[\cdot ; \cdot], f_{j}(t) \in \operatorname{supp} \mu[\cdot ; t]$ п.в. и $d(\mu[\cdot ; t], \nu[\cdot ; t])<\varepsilon$ n.в., где

$$
\nu[\cdot ; t] \doteq \frac{1}{N} \sum_{j=1}^{N} \delta_{f_{j}(t)}[\cdot], \quad t \in \mathbb{R} .
$$

(Из лемми 4 вытекает, ито $\nu[\cdot ; \cdot] \in S^{c}\left(\mathbb{R}, \mathscr{M}_{\tau}\right)$ u $\operatorname{Mod} \nu[\cdot ; \cdot] \subset \sum_{j=1}^{N} \operatorname{Mod} f_{j} \subset$ $\operatorname{Mod} \mu[\cdot ; \cdot]$.)

Доказательство теоремы 8 приведено в $\S 5$.

СлЕДСТВИЕ 8.1. Пусть $(\mathscr{U}, \rho)$ - полное метрическое пространство, $\mu[\cdot ; \cdot]$ $\in S^{c}\left(\mathbb{R}, \mathscr{M}_{\tau}\right)$. Тогда найдутся функции $f_{j} \in S^{c}(\mathbb{R}, \mathscr{U}), j \in \mathbb{N}$, такие, что $\operatorname{Mod} f_{j} \subset \operatorname{Mod} \mu[\cdot ; \cdot], f_{j}(t) \in \operatorname{supp} \mu[\cdot ; t]$ n.в. $u$

$$
\lim _{L \rightarrow \infty} D_{\infty}^{(d)}\left(\mu[\cdot ; \cdot], \frac{1}{L} \sum_{j=1}^{L} \delta_{f_{j}(\cdot)}[\cdot]\right)=0 .
$$

ДокАЗАТЕЛЬСТво. Для каждого числа $\varepsilon=1 / i, i \in \mathbb{N}$, найдем в соответствии с теоремой 8 числа $N(i) \in \mathbb{N}$ и функции $f_{j}^{(i)} \in S^{c}(\mathbb{R}, \mathscr{U}), j=1, \ldots, N(i)$. Выберем натуральные числа $m_{i}, i \in \mathbb{N}$, так, что

$$
m_{i} \frac{N(i)}{N(i+1)} \rightarrow \infty
$$

при $i \rightarrow \infty$. Каждое число $j \in \mathbb{Z}^{+}$можно однозначно представить в виде

$$
j=\sum_{i=1}^{s} m_{i} N(i)+n N(s+1)+k
$$


где $s=s(j) \in \mathbb{Z}^{+}, n=n(j) \in\left\{0, \ldots, m_{s+1}-1\right\}, k=k(j) \in\{0, \ldots, N(s+1)-1\}$ (первая сумма в представлении (5) отсутствует, если $j<m_{1} N(1)$, в этом случае $s=s(j)=0)$. Положим

$$
f_{j}(\cdot)=f_{k(j)+1}^{(s(j)+1)}(\cdot), \quad j \in \mathbb{N} .
$$

Для любого $L \in \mathbb{N}$ справедливо неравенство

$$
\begin{aligned}
D_{\infty}^{(d)}\left(\mu[\cdot ; \cdot], \frac{1}{L} \sum_{j=1}^{L}\right. & \left.\delta_{f_{j}(\cdot)}[\cdot]\right) \\
& \leqslant \frac{1}{L}\left(\sum_{i=1}^{s(L)} \frac{1}{i} m_{i} N(i)+\left(\frac{n(L)}{s(L)+1}+1\right) N(s(L)+1)\right) .
\end{aligned}
$$

Так как $L \geqslant m_{s(L)} N(s(L))$ (при $L \geqslant m_{1} N(1)$ ), то из неравенства (6) и выбора чисел $m_{i}, i \in \mathbb{N}$, следует (4). Следствие 8.1 доказано.

СлЕДСТВИЕ 8.2. Пусть $(\mathscr{U}, \rho)$ - полное метрическое пространство, $\mu[\cdot ; \cdot] \in S\left(\mathbb{R}, \mathscr{M}_{\tau}\right) . \quad$ Тогда существуют последовательности множеств $T_{i} \subset \mathbb{R}, i \in \mathbb{N}$, и функций $f_{j} \in S(\mathbb{R}, \mathscr{U}), j \in \mathbb{N}$, такие, ито $\left\{T_{i}\right\} \in \mathfrak{M}(\operatorname{Mod} \mu[\cdot ; \cdot])$, $\operatorname{Mod} f_{j} \subset \operatorname{Mod} \mu[\cdot ; \cdot], f_{j}(t) \in \operatorname{supp} \mu[\cdot ; t]$ n.в. и для всех $i \in \mathbb{N}$

$$
\lim _{L \rightarrow \infty} \underset{t \in T_{i}}{\operatorname{esssup}} d\left(\mu[\cdot ; t], \frac{1}{L} \sum_{j=1}^{L} \delta_{f_{j}(t)}[\cdot]\right)=0 .
$$

ЗАмечАниЕ. Так как $\varkappa\left(\bigcup_{i \leqslant l} T_{i}\right) \rightarrow 0$ при $l \rightarrow \infty$, то из (7) следует, что

$$
D^{(d)}\left(\mu[\cdot ; \cdot], \frac{1}{L} \sum_{j=1}^{L} \delta_{f_{j}(\cdot)}[\cdot]\right) \rightarrow 0
$$

при $L \rightarrow \infty$.

ДокАЗАТЕЛЬСТво СЛЕДСТвИя 8.2. Из теоремы 1 следует существование множеств $T_{i} \subset \mathbb{R}, i \in \mathbb{N}$, таких, что $\left\{T_{i}\right\} \in \mathfrak{M}(\operatorname{Mod} \mu[\cdot ; \cdot])$, мерозначная функция $\mu[\cdot ; t]$ определена при всех $t \in \bigcup_{i} T_{i}$ и $\bigcup_{t \in T_{i}} \mu[\cdot ; t]$ - предкомпактные множества в метрическом пространстве $\left(\mathscr{M}_{\tau}, d\right)$. Для всех $l \in \mathbb{N}$ определим функции

$$
\mu_{l}[\cdot ; t]= \begin{cases}\mu[\cdot ; t], & \text { если } t \in \bigcup_{i \leqslant l} T_{i}, \\ \delta_{x_{0}}[\cdot], & \text { если } t \notin \bigcup_{i \leqslant l} T_{i} .\end{cases}
$$

Тогда (в силу леммы 2) $\mu_{l}[\cdot ; \cdot] \in S^{c}\left(\mathbb{R}, \mathscr{M}_{\tau}\right)$ и $\operatorname{Mod} \mu_{l}[\cdot ; \cdot] \subset \operatorname{Mod} \mu[\cdot ; \cdot]$. Из теоремы 8 для любого $l \in \mathbb{N}$ вытекает сушествование чисел $N(l) \in \mathbb{N}$ и функций $f_{j}^{(l)} \in S(\mathbb{R}, \mathscr{U}), j=1, \ldots, N(l)$, таких, что $\operatorname{Mod} f_{j}^{(l)} \subset \operatorname{Mod} \mu_{l}[\cdot ; \cdot]$, $f_{j}^{(l)}(t) \in \operatorname{supp} \mu_{l}[\cdot ; t]$ п.в. и

$$
d\left(\mu_{l}[\cdot ; t], \frac{1}{N(l)} \sum_{j=1}^{N(l)} \delta_{f_{j}^{(l)}(t)}[\cdot]\right)<\frac{1}{l} \text { п.в. }
$$


Так как числа $N(l)$ всегда можно заменить на кратные, то без ограничения общности будем считать, что $N(l+1) \geqslant N(l)$ при всех $l \in \mathbb{N}$. Определим функции

$$
g_{j}^{(l)}(t)= \begin{cases}f_{j}^{(l)}(t), & \text { если } t \in \bigcup_{i \leqslant l} T_{i}, \\ f_{j}^{(i)}(t), & \text { если } t \in T_{i}, i>l,\end{cases}
$$

$l \in \mathbb{N}, j=1, \ldots, N(l)$. В силу леммы 2 имеем $g_{j}^{(l)} \in S(\mathbb{R}, \mathscr{U})$ и $\operatorname{Mod} g_{j}^{(l)} \subset$ $\operatorname{Mod} \mu[\cdot ; \cdot]$, при этом $g_{j}^{(l)}(t) \in \operatorname{supp} \mu[\cdot ; t]$ п.в. Выберем натуральные числа $m_{l}$, $l \in \mathbb{N}$, так, что

$$
m_{l} \frac{N(l)}{N(l+1)} \rightarrow \infty
$$

при $l \rightarrow \infty$. Каждому числу $j \in \mathbb{N}$ в представлении (5) однозначно соответствуют числа $s=s(j)$ и $k=k(j)$. Положим

$$
f_{j}(t)=g_{k(j)+1}^{(s(j)+1)}(t), \quad t \in \mathbb{R}
$$

Функции $f_{j}$ обладают всеми требуемыми свойствами, при этом равенство $(7)$ доказывается так же, как и равенство (4) при доказательстве следствия 8.1. Следствие 8.2 доказано.

\section{§4. Доказательство теоремы 4}

Лемма 5. Пусть $\mu[\cdot ; \cdot] \in S\left(\mathbb{R}, \mathscr{M}_{\tau}\right), \mathscr{F}(\cdot, \cdot) \in S\left(\mathbb{R}, C_{b}(\mathscr{U})\right), \mathscr{F}(\cdot, t) \in C_{b}^{+}(\mathscr{U})$ n.в. и $\mu(\mathscr{F}(\cdot, t) ; t) \geqslant \varepsilon>0$ n.в. Тогда $\mu^{\mathscr{F}(\cdot, \cdot)}[\cdot ; \cdot] \in S\left(\mathbb{R}, \mathscr{M}_{\tau}\right) u \operatorname{Mod} \mu^{\mathscr{F}(\cdot, \cdot)}[\cdot ; \cdot]$ $\subset \operatorname{Mod} \mu[\cdot ; \cdot]+\operatorname{Mod} \mathscr{F}(\cdot, \cdot) . \quad$ Eсли, кроме того, $\mu[\cdot ; \cdot] \in S^{c}\left(\mathbb{R}, \mathscr{M}_{\tau}\right) \quad$ u $\mathscr{F}(\cdot, \cdot) \in S^{c}\left(\mathbb{R}, C_{b}(\mathscr{U})\right)$, mо также $\mu^{\mathscr{F}(\cdot, \cdot)}[\cdot ; \cdot] \in S^{c}\left(\mathbb{R}, \mathscr{M}_{\tau}\right)$.

ДокАЗАТЕльство. Функция $t \mapsto(\mu[\cdot ; t], \mathscr{F}(\cdot, t)) \in\left(\mathscr{M}_{\tau} \times C_{b}(\mathscr{U}), D^{0}\right), t \in \mathbb{R}$, почти периодична по Степанову и $\operatorname{Mod}(\mu[\cdot ; \cdot], \mathscr{F}(\cdot, \cdot))=\operatorname{Mod} \mu[\cdot ; \cdot]+\operatorname{Mod} \mathscr{F}(\cdot, \cdot)$. Обозначим $P(\varepsilon)=\left\{(\mu, \mathscr{F}) \in \mathscr{M}_{\tau} \times C_{b}(\mathscr{U}): \mathscr{F} \in C_{b}^{+}(\mathscr{U}), \mu(\mathscr{F}) \geqslant \varepsilon\right\}$. Так как множество $P(\varepsilon)$ замкнуто в полном метрическом пространстве $\left(\mathscr{M}_{\tau} \times C_{b}(\mathscr{U}), D^{0}\right)$, $(\mu[\cdot ; t], \mathscr{F}(\cdot, t)) \in P(\varepsilon)$ п.в. и отображение $\mathscr{M}_{\tau} \times C_{b}(\mathscr{U}) \ni(\mu, \mathscr{F}) \mapsto \mu^{\mathscr{F}} \in \mathscr{M}_{\tau}$ непрерывно на множестве $P(\varepsilon)$, то в силу следствия леммы 3 получаем $\mu^{\mathscr{F}(\cdot, \cdot)}[\cdot ; \cdot] \in$ $S\left(\mathbb{R}, \mathscr{M}_{\tau}\right)$ и $\operatorname{Mod} \mu^{\mathscr{F}(\cdot, \cdot)}[\cdot ; \cdot] \subset \operatorname{Mod}(\mu[\cdot ; \cdot], \mathscr{F}(\cdot, \cdot))=\operatorname{Mod} \mu[\cdot ; \cdot]+\operatorname{Mod} \mathscr{F}(\cdot, \cdot)$. Пусть теперь $\mu[\cdot ; \cdot] \in S^{c}\left(\mathbb{R}, \mathscr{M}_{\tau}\right)$ и $\mathscr{F}(\cdot, \cdot) \in S^{c}\left(\mathbb{R}, C_{b}(\mathscr{U})\right)$. Найдутся компактные множества $\mathscr{K} \subset \mathscr{M}_{\tau}$ и $\mathscr{C} \subset C_{b}(\mathscr{U})$ такие, что $\mu[\cdot ; t] \in \mathscr{K}$ п.в. и $\mathscr{F}(\cdot, t) \in \mathscr{C}$ п.в. Тогда множество $(\mathscr{K} \times \mathscr{C}) \cap P(\varepsilon)$ и, следовательно, его образ при непрерывном отображении $(\mu, \mathscr{F}) \mapsto \mu^{\mathscr{F}}$, которому при п.в. $t \in \mathbb{R}$ принадлежат меры $\mu^{\mathscr{F}(\cdot, t)}[\cdot ; t]$, также компактны. Лемма доказана.

Лемма 6. Пусть $\mu[\cdot ; \cdot] \in S^{c}\left(\mathbb{R}, \mathscr{M}_{\tau}\right)$. Тогда для любого $\delta>0$ найдутся число $\varepsilon>0$ и функиия $t \mapsto \mathscr{F}(\cdot, t) \in C_{b}^{+}(\mathscr{U}), t \in \mathbb{R}$, из пространства $S^{c}\left(\mathbb{R}, C_{b}(\mathscr{U})\right)$ maкuе, umo $\operatorname{Mod} \mathscr{F}(\cdot, \cdot) \subset \operatorname{Mod} \mu[\cdot ; \cdot], \mu(\mathscr{F}(\cdot, t) ; t) \geqslant \varepsilon$ n.в. u diamsupp $\mathscr{F}(\cdot, t) \leqslant \delta$ n.в. 
ДоказАТЕЛЬСтво. Сушествует предкомпактное множество $\mathscr{K} \subset\left(\mathscr{M}_{\tau}, d\right)$ такое, что $\mu[\cdot ; t] \in \mathscr{K}$ п.в. Пусть $K \subset \mathscr{U}$ - компакт, для которого $\mu[K]>\frac{1}{2}$ для всех мер $\mu \in \mathscr{K}$. Выберем точки $x_{i} \in K, i=1, \ldots, N$, образующие конечную $\delta / 4$-сеть для множества $K$. Обозначим

$$
\Phi_{i}(\cdot)=\theta\left(2-\frac{4}{\delta} \rho\left(\cdot, x_{i}\right)\right) \in C_{b}^{+}(\mathscr{U}),
$$

$g_{i}(t)=\mu\left(\Phi_{i} ; t\right), t \in \mathbb{R}, i=1, \ldots, N . \quad$ Тогда $g_{i}(t) \geqslant 0$ п.в., $g_{i} \in S(\mathbb{R}, \mathbb{R})$ и $\operatorname{Mod} g_{i} \subset \operatorname{Mod} \mu[\cdot ; \cdot] . \quad$ Так как $\sum_{i=1}^{N} \Phi_{i}(x) \geqslant 1$ для всех $x \in K$, то $\sum_{i=1}^{N} g_{i}(t) \geqslant \frac{1}{2}$ п.в. В пространстве $\mathbb{R}^{N}$ введем норму $\|y\|_{N}=\max _{i=1, \ldots, N}\left|y_{i}\right|$, $y=\left(y_{1}, \ldots, y_{N}\right) \in \mathbb{R}^{N}$. Обозначим через $g(\cdot): \mathbb{R} \rightarrow \mathbb{R}^{N}$ вектор-функцию с компонентами $g_{i}(\cdot), i=1, \ldots, N$. Имеем $g(\cdot) \in S\left(\mathbb{R},\left(\mathbb{R}^{N},\|\cdot\|_{N}\right)\right)$ и $\operatorname{Mod} g=\sum_{i} \operatorname{Mod} g_{i} \subset \operatorname{Mod} \mu[\cdot ; \cdot]$. В силу теоремы 2 найдутся последовательность $\left\{T_{j}\right\} \in \mathfrak{M}(\operatorname{Mod} g)$ и точки $y^{(j)} \in \mathbb{R}^{N}, j \in \mathbb{N}$, такие, что $\left\|y^{(j)}-g(t)\right\|_{N} \leqslant 1 /(8 N)$ при всех $j \in \mathbb{N}$ и п.в. $t \in T_{j}$. Каждому индексу $j$ поставим в соответствие число $i(j) \in\{1, \ldots, N\}$ так, что $y_{i(j)}^{(j)}=\max _{i=1, \ldots, N} y_{i}^{(j)}$. Тогда $y_{i(j)}^{(j)} \geqslant 3 /(8 N)$ (если mes $\left.T_{j} \neq 0\right)$ и, следовательно, $g_{i(j)}(t) \geqslant 1 /(4 N)$ при всех $j \in \mathbb{N}$ и п.в. $t \in T_{j}$. Положим

$$
\mathscr{F}(\cdot, t)=\sum_{j} \Phi_{i(j)}(\cdot) \chi_{T_{j}}(t), \quad t \in \mathbb{R} .
$$

Из леммы 2 следует, что $\mathscr{F}(\cdot, \cdot) \in S\left(\mathbb{R}, C_{b}(\mathscr{U})\right)$ и $\operatorname{Mod} \mathscr{F}(\cdot, \cdot) \subset \operatorname{Mod} \mu[\cdot ; \cdot]$. Более того, так как функция $t \mapsto \mathscr{F}(\cdot, t), t \in \mathbb{R}$, принимает конечное число значений, то $\mathscr{F}(\cdot, \cdot) \in S^{c}\left(\mathbb{R}, C_{b}(\mathscr{U})\right)$. При п.в. $t \in \mathbb{R}$ выполняется включение $\mathscr{F}(\cdot, t) \in C_{b}^{+}(\mathscr{U})$, при этом при всех $j \in \mathbb{N}$ и п.в. $t \in T_{j}$ имеем $\operatorname{supp} \mathscr{F}(\cdot, t) \subset K\left(x_{i(j)}, \delta / 2\right)$ и $\mu(\mathscr{F}(\cdot, t) ; t)=g_{i(j)}(t) \geqslant 1 /(4 N) \doteq \varepsilon$. Лемма доказана.

TEОРема 9. Пусть $(\mathscr{U}, \rho)$ - полное метрическое пространство, $\mu[\cdot ; \cdot] \in$ $S^{c}\left(\mathbb{R}, \mathscr{M}_{\tau}\right)$. Тогда существует функиия $f \in S^{c}(\mathbb{R}, \mathscr{U})$ такая, ито $\operatorname{Mod} f \subset$ $\operatorname{Mod} \mu[\cdot ; \cdot]$ u $f(t) \in \operatorname{supp} \mu[\cdot ; t]$ n.в.

ДоКАЗАТЕЛЬСТво. Обозначим $\mu_{0}[\cdot ; \cdot]=\mu[\cdot ; \cdot]$. Будем последовательно при $j=1,2, \ldots$ находить функции $\mathscr{F}_{j}(\cdot, \cdot) \in S^{c}\left(\mathbb{R}, C_{b}(\mathscr{U})\right)$ и $\mu_{j}[\cdot ; \cdot] \in S^{c}\left(\mathbb{R}, \mathscr{M}_{\tau}\right)$, для которых $\operatorname{Mod} \mathscr{F}_{j}(\cdot, \cdot) \subset \operatorname{Mod} \mu[\cdot ; \cdot]$ и $\operatorname{Mod} \mu_{j}[\cdot ; \cdot] \subset \operatorname{Mod} \mu[\cdot ; \cdot]$. Предположим, что они уже найдены для некоторого $j \in \mathbb{Z}^{+}$(если $j=0$, то определяются только функция $\left.\mu_{0}[\cdot ; \cdot]\right)$. Для мерозначной функции $\mu_{j}[\cdot ; \cdot]$ выберем в соответствии с леммой 6 функцию $t \mapsto \mathscr{F}_{j+1}(\cdot, t) \in C_{b}^{+}(\mathscr{U}), t \in \mathbb{R}$, такую, что $\mathscr{F}_{j+1}(\cdot, \cdot) \in S^{c}\left(\mathbb{R}, C_{b}(\mathscr{U})\right), \operatorname{Mod} \mathscr{F}_{j+1}(\cdot, \cdot) \subset \operatorname{Mod} \mu_{j}[\cdot ; \cdot] \subset \operatorname{Mod} \mu[\cdot ; \cdot]$, $\mu_{j}\left(\mathscr{F}_{j+1}(\cdot, t) ; t\right) \geqslant \varepsilon_{j+1}>0$ и $\operatorname{diamsupp} \mathscr{F}_{j+1}(\cdot, t) \leqslant 2^{-j-1}$ п.в. Положим $\mu_{j+1}[\cdot ; \cdot]=\left(\mu_{j}\right)^{\mathscr{F}_{j+1}(\cdot, \cdot)}[\cdot ; \cdot] . \quad$ В силу леммы $5 \mu_{j+1}[\cdot ; \cdot] \in S^{c}\left(\mathbb{R}, \mathscr{M}_{\tau}\right)$ и $\operatorname{Mod} \mu_{j+1}[\cdot ; \cdot] \subset \operatorname{Mod} \mu_{j}[\cdot ; \cdot]+\operatorname{Mod} \mathscr{F}_{j+1}(\cdot, \cdot) \subset \operatorname{Mod} \mu[\cdot ; \cdot] . \quad$ При этом $\operatorname{supp} \mu_{j+1}[\cdot ; t] \subset \operatorname{supp} \mu_{j}[\cdot ; t] \cap \operatorname{supp} \mathscr{F}_{j+1}(\cdot, t)$ п.в., поэтому $\operatorname{supp} \mu_{j+1}[\cdot ; t] \subset$ $\operatorname{supp} \mu_{j}[\cdot ; t] \subset \operatorname{supp} \mu[\cdot ; t] \quad$ и $\operatorname{diam} \operatorname{supp} \mu_{j+1}[\cdot ; t] \leqslant 2^{-j-1}$ п.в. Продолжим неограниченно нахождение функций $\mathscr{F}_{j}(\cdot, \cdot)$ и $\mu_{j}[\cdot ; \cdot]$.

Множество $\bigcap_{j} \operatorname{supp} \mu_{j}[\cdot ; t]$ п.в. состоит из одной точки $[15$, c. 40], которую обозначим $f(t)$, при этом $f(t) \in \operatorname{supp} \mu[\cdot ; t]$. При всех $j \in \mathbb{N}$ и п.в. $t \in \mathbb{R}$ справедливо неравенство $d\left(\mu_{j}[\cdot ; t], \delta_{f(t)}[\cdot]\right) \leqslant \operatorname{diam} \operatorname{supp} \mu_{j}[\cdot ; t] \leqslant 2^{-j}$, следовательно, $\delta_{f(\cdot)} \in M\left(\mathbb{R}, \mathscr{M}_{\tau}\right)$. Так как $D_{\infty}^{(d)}\left(\mu_{j}[\cdot ; \cdot], \delta_{f(\cdot)}[\cdot]\right) \rightarrow 0$ при $j \rightarrow \infty$ и 
$\mu_{j}[\cdot ; \cdot] \in S^{c}\left(\mathbb{R}, \mathscr{M}_{\tau}\right), j \in \mathbb{N}$, то $\delta_{f(\cdot)} \in S^{c}\left(\mathbb{R}, \mathscr{M}_{\tau}\right)$ и $\operatorname{Mod} \delta_{f(\cdot)} \subset \operatorname{Mod} \mu[\cdot ; \cdot]$. Отсюда $f \in S^{c}(\mathbb{R}, \mathscr{U})$ и $\operatorname{Mod} f \subset \operatorname{Mod} \mu[\cdot ; \cdot]$. Теорема 9 доказана.

Зафиксируем числа $\varepsilon_{1} \in(0,1)$ и $\varepsilon_{2} \in\left(\varepsilon_{1}, 1\right)$. Пусть $(0,1) \ni \lambda \mapsto \omega(\lambda) \in \mathbb{R}$-неотрицательная непрерывная функция такая, что $\operatorname{supp} \omega(\cdot) \subset\left[\varepsilon_{1}, \varepsilon_{2}\right]$ и $\int_{0}^{1} \omega(\lambda) d \lambda=1$. Функция $\lambda \mapsto r_{\lambda}(\mu, x)$ при всех $\mu \in \mathscr{M}_{\tau}$ и $x \in \mathscr{U}$ непрерьвна слева и не убывает на интервале $(0,1)$. Обозначим

$$
G(\mu, x)=\int_{0}^{1} \omega(\lambda) r_{\lambda}(\mu, x) d \lambda
$$

$\mu \in \mathscr{M}_{\tau}, x \in \mathscr{U}$.

Рассмотрим полное метрическое пространство $\left(\mathscr{M}_{\tau} \times \mathscr{U}, R\right)$ с метрикой

$$
R((\mu, x),(\nu, y))=d(\mu, \nu)+\rho(x, y),
$$

где $(\mu, x),(\nu, y) \in \mathscr{M}_{\tau} \times \mathscr{U}$.

ЛЕмма 7. Функция $G(\cdot, \cdot)$ непрерывна на метрическом пространстве $\left(\mathscr{M}_{\tau} \times \mathscr{U}, R\right)$.

ДоказАТЕЛьство. Для любых $r \geqslant 0, x, y \in \mathscr{U}$ имеем

$$
K(x, r) \subset K(y, r+\rho(x, y)) \text { и } K(y, r) \subset K(x, r+\rho(x, y)),
$$

поэтому $\left|r_{\lambda}(\mu, x)-r_{\lambda}(\mu, y)\right| \leqslant \rho(x, y), \lambda \in(0,1), \mu \in \mathscr{M}_{\tau}$, и, следовательно,

$$
|G(\mu, x)-G(\mu, y)| \leqslant \rho(x, y)
$$

для всех $\mu \in \mathscr{M}_{\tau}$.

Зафиксируем меру $\mu \in \mathscr{M}_{\tau}$. Пусть $\varepsilon^{\prime} \in\left(\varepsilon_{2}, 1\right), \nu \in \mathscr{M}_{\tau}$ и $d(\mu, \nu)<$ $\min \left\{\varepsilon_{1}, \varepsilon^{\prime}-\varepsilon_{2}\right\}$. Выберем любое число $\delta \in\left(d(\mu, \nu), \min \left\{\varepsilon_{1}, 1-\varepsilon_{2}\right\}\right)$. В силу определения метрики $d$ имеем $\mu[K(x, r+\delta)] \geqslant \mu\left[(K(x, r))^{\delta}\right]>\nu[K(x, r)]-\delta$ для всех $x \in \mathscr{U}, r \geqslant 0$, поэтому

$$
r_{\lambda-\delta}(\mu, x) \leqslant r_{\lambda}(\nu, x)+\delta
$$

$\lambda \in\left[\varepsilon_{1}, 1\right]$. Обозначим $C_{\omega}(\gamma)=\max _{\left|\lambda^{\prime}-\lambda^{\prime \prime}\right| \leqslant \gamma}\left|\omega\left(\lambda^{\prime}\right)-\omega\left(\lambda^{\prime \prime}\right)\right|, \gamma \geqslant 0$. Из (10) следует неравенство

$$
\begin{aligned}
G(\mu, x)-C_{\omega}(\delta) r_{\varepsilon_{2}}(\mu, x) & \leqslant \int_{0}^{1-\delta} \omega(\lambda+\delta) r_{\lambda}(\mu, x) d \lambda=\int_{0}^{1} \omega(\lambda) r_{\lambda-\delta}(\mu, x) d \lambda \\
& \leqslant \delta+\int_{0}^{1} \omega(\lambda) r_{\lambda}(\nu, x) d \lambda=\delta+G(\nu, x),
\end{aligned}
$$

из которого при $\delta \rightarrow d(\mu, \nu)$ получаем

$$
G(\mu, x)-G(\nu, x) \leqslant d(\mu, \nu)+C_{\omega}(d(\mu, \nu)) r_{\varepsilon_{2}}(\mu, x) .
$$


В неравенстве (10) поменяем местами меры $\mu$ и $\nu$. Тогда при $\delta \rightarrow d(\mu, \nu)$, в частности, имеем

$$
r_{\varepsilon_{2}}(\nu, x) \leqslant r_{\varepsilon^{\prime}}(\mu, x)+d(\mu, \nu) .
$$

Аналогично неравенству (11), учитывая (12), получаем

$$
G(\nu, x)-G(\mu, x) \leqslant d(\mu, \nu)+\left(r_{\varepsilon^{\prime}}(\mu, x)+d(\mu, \nu)\right) C_{\omega}(d(\mu, \nu)) .
$$

Следовательно,

$$
|G(\mu, x)-G(\nu, x)| \leqslant d(\mu, \nu)+\left(r_{\varepsilon}^{\prime}(\mu, x)+d(\mu, \nu)\right) C_{\omega}(d(\mu, \nu)) .
$$

Из последнего неравенства вытекает непрерывность функции $G(\nu, x)$ (при фиксированном $x \in \mathscr{U})$ в точке $\nu=\mu \in \mathscr{M}_{\tau}$. Вместе с неравенством (9) это доказывает непрерывность функции $G(\cdot, \cdot)$ на метрическомпространстве $\left(\mathscr{M}_{\tau} \times \mathscr{U}, R\right)$. Лемма доказана.

Лемma 8. Eсли $\mu[\cdot ; \cdot] \in S^{c}\left(\mathbb{R}, \mathscr{M}_{\tau}\right)$ u $g \in S^{c}(\mathbb{R}, \mathscr{U})$, mo $G(\mu[\cdot ; \cdot], g(\cdot)) \in$ $S(\mathbb{R}, \mathbb{R}) \cap L^{\infty}(\mathbb{R}, \mathbb{R}) u \operatorname{Mod} G(\mu[\cdot ; \cdot], g(\cdot)) \subset \operatorname{Mod} \mu[\cdot ; \cdot]+\operatorname{Mod} g$.

ДокаЗАТЕльство. Функция $t \mapsto(\mu[\cdot ; t], g(t)) \in \mathscr{M}_{\tau} \times \mathscr{U}, t \in \mathbb{R}$, принадлежит пространству $S\left(\mathbb{R},\left(\mathscr{M}_{\tau} \times \mathscr{U}, R\right)\right)$ и $\operatorname{Mod}(\mu[\cdot ; \cdot], g(\cdot))=\operatorname{Mod} \mu[\cdot ; \cdot]+\operatorname{Mod} g$. Пусть $\mathscr{K} \subset \mathscr{M}_{\tau}$ и $K \subset \mathscr{U}$ - компактные множества такие, что $\mu[\cdot ; t] \in \mathscr{K}$ и $g(t) \in K$ п.в. Тогда $\mathscr{K} \times K$ - компакт в метрическом пространстве $\left(\mathscr{M}_{\tau} \times \mathscr{U}, R\right)$ и $(\mu[\cdot ; t], g(t)) \in \mathscr{K} \times K$ п.в. В силу ограниченности функции $G(\cdot, \cdot)$ на множестве $\mathscr{K} \times K$ получаем $G(\mu[\cdot ; \cdot], g(\cdot)) \in L^{\infty}(\mathbb{R}, \mathbb{R})$. Наконец, из леммы 7 и следствия леммы 3 вытекает, что $G(\mu[\cdot ; \cdot], g(\cdot)) \in S(\mathbb{R}, \mathbb{R})$ и $\operatorname{Mod} G(\mu[\cdot ; \cdot], g(\cdot)) \subset$ $\operatorname{Mod}(\mu[\cdot ; \cdot], g(\cdot))=\operatorname{Mod} \mu[\cdot ; \cdot]+\operatorname{Mod} g$. Лемма доказана.

Определим функцию

$$
\omega(\lambda)= \begin{cases}0, & \text { если } \lambda \in\left(0, \frac{\varepsilon}{2}\right) \cup(\varepsilon, 1), \\ 16 \varepsilon^{-2}\left(\lambda-\frac{\varepsilon}{2}\right), & \text { если } \lambda \in\left[\frac{\varepsilon}{2}, \frac{3 \varepsilon}{4}\right], \\ 16 \varepsilon^{-2}(\varepsilon-\lambda), & \text { если } \lambda \in\left(\frac{3 \varepsilon}{4}, \varepsilon\right]\end{cases}
$$

и соответствующую ей функцию $G(\cdot, \cdot)$ (см. (8)). Тогда

$$
r_{\varepsilon / 2}(\mu, x) \leqslant G(\mu, x) \leqslant r_{\varepsilon}(\mu, x)
$$

при всех $\mu \in \mathscr{M}_{\tau}$ и $x \in \mathscr{U}$. Положим

$$
Y(r, y ; x)=\theta\left(\frac{2}{\varepsilon}\left(r+\frac{\varepsilon}{2}-\rho(x, y)\right)\right)
$$

$r \in \mathbb{R}, x, y \in \mathscr{U} ; Y(r, y ; \cdot) \in C_{b}^{+}(\mathscr{U})$ и функция $\mathbb{R} \times \mathscr{U} \ni(r, y) \mapsto Y(r, y ; \cdot) \in C_{b}(\mathscr{U})$ непрерывна и ограничена на полном метрическом пространстве $\left(\mathbb{R} \times \mathscr{U}, R^{\prime}\right)$, где

$$
R^{\prime}\left(\left(r_{1}, y_{1}\right),\left(r_{2}, y_{2}\right)\right)=\left|r_{1}-r_{2}\right|+\rho\left(y_{1}, y_{2}\right)
$$


$r_{1}, r_{2} \in \mathbb{R}, y_{1}, y_{2} \in \mathscr{U} . \quad$ Так как $G(\mu[\cdot ; \cdot], g(\cdot)) \in S(\mathbb{R}, \mathbb{R}) \cap L^{\infty}(\mathbb{R}, \mathbb{R})$ и $g \in S^{c}(\mathbb{R}, \mathscr{U})$, то, используя следствие леммы 3 , получаем, что функция $t \mapsto \Theta(\cdot, t) \doteq Y(G(\mu[\cdot ; t], g(t)), g(t) ; \cdot), \quad t \in \mathbb{R}$, принадлежит пространству $S^{c}\left(\mathbb{R}, C_{b}(\mathscr{U})\right)$ и

$$
\operatorname{Mod} \Theta(\cdot, \cdot) \subset \operatorname{Mod} G(\mu[\cdot ; \cdot], g(\cdot))+\operatorname{Mod} g \subset \operatorname{Mod} \mu[\cdot ; \cdot]+\operatorname{Mod} g .
$$

При этом $\Theta(\cdot, t) \in C_{b}^{+}(\mathscr{U})$ п.в. Так как

$$
\mu(\Theta(\cdot, t) ; t) \geqslant \mu\left[K\left(g(t), r_{\varepsilon / 2}(\mu[\cdot, t], g(t))\right) ; t\right] \geqslant \frac{\varepsilon}{2} \text { п.в. }
$$

то в силу леммы 5 получаем $\mu^{\Theta(\cdot, \cdot)}[\cdot ; \cdot] \in S^{c}\left(\mathbb{R}, \mathscr{M}_{\tau}\right)$ и $\operatorname{Mod} \mu^{\Theta(\cdot, \cdot)}[\cdot ; \cdot] \subset$ $\operatorname{Mod} \mu[\cdot ; \cdot]+\operatorname{Mod} \Theta(\cdot, \cdot) \subset \operatorname{Mod} \mu[\cdot ; \cdot]+\operatorname{Mod} g . \quad$ Более того, $\operatorname{supp} \Theta(\cdot, t) \subset$ $K(g(t), G(\mu[\cdot ; t], g(t))+\varepsilon / 2)$ п.в., поэтому $\operatorname{supp} \mu^{\Theta(\cdot, t)}[\cdot ; t] \subset \operatorname{supp} \mu[\cdot ; t] \cap$ $K\left(g(t), r_{\varepsilon}(\mu[\cdot ; t], g(t))+\varepsilon / 2\right)$ п.в. В соответствии с теоремой 9 найдется функция $f \in S^{c}(\mathbb{R}, \mathscr{U})$ такая, что $\operatorname{Mod} f \subset \operatorname{Mod} \mu^{\Theta(\cdot, \cdot)}[\cdot ; \cdot]$ и $f(t) \in \operatorname{supp} \mu^{\Theta(\cdot, t)}[\cdot ; t]$ п.в. Тогда $f(t) \in \operatorname{supp} \mu[\cdot ; t]$ п.в. и $\rho(f(t), g(t))<r_{\varepsilon}(\mu[\cdot ; t], g(t))+\varepsilon$ п.в. Функция $f$ обладает всеми требуемыми свойствами. Теорема 4 доказана.

\section{§5. Доказательство теоремы 8}

ЛЕмма 9. Пусть $\mu[\cdot ; \cdot] \in S^{c}\left(\mathbb{R}, \mathscr{M}_{\tau}\right)$,

$$
\nu[\cdot ; t]=\sum_{j=1}^{N} \lambda_{j}(t) \delta_{x_{j}(t)}[\cdot], \quad t \in \mathbb{R},
$$

где $N \in \mathbb{N}, \lambda_{j}(t) \geqslant 0, \sum_{j=1}^{N} \lambda_{j}(t)=1$ n.в., $\lambda_{j}(\cdot) \in S(\mathbb{R}, \mathbb{R}), x_{j}(\cdot) \in S^{c}(\mathbb{R}, \mathscr{U})$ u $d(\mu[\cdot ; t], \nu[\cdot ; t])<\varepsilon$ n.в., $\varepsilon>0 . \quad$ Тогда для любого $\varepsilon^{\prime}>\varepsilon$ существуют функиии $g_{j} \in S^{c}(\mathbb{R}, \mathscr{U}), j=1, \ldots, N$, такие, что $\operatorname{Mod} g_{j} \subset \operatorname{Mod} \mu[\cdot ; \cdot]+$ $\operatorname{Mod} x_{j}(\cdot), g_{j}(t) \in \operatorname{supp} \mu[\cdot ; t]$ n.в. $u d\left(\nu^{\prime}[\cdot ; t], \nu[\cdot ; t]\right)<\varepsilon^{\prime}$ n.в., zде $\nu^{\prime}[\cdot ; t]=$ $\sum_{j=1}^{N} \lambda_{j}(t) \delta_{g_{j}(t)}[\cdot], t \in \mathbb{R}$. (Из леммы 4 вытекает, ито $\nu^{\prime}[\cdot ; \cdot] \in S^{c}\left(\mathbb{R}, \mathscr{M}_{\tau}\right) u$ $\operatorname{Mod} \nu^{\prime}[\cdot ; \cdot] \subset \operatorname{Mod} \mu[\cdot ; \cdot]+\sum_{j=1}^{N} \operatorname{Mod} x_{j}(\cdot)+\sum_{j=1}^{N} \operatorname{Mod} \lambda_{j}(\cdot)$.)

ДокаЗАТЕЛЬСТво. Пусть мерозначные функции $\mu[\cdot ; t]$ и $\nu[\cdot ; t]$ определены и $d(\mu[\cdot ; t], \nu[\cdot ; t])<\varepsilon$ при всех $t \in T$, где $T \subset \mathbb{R}$ и mes $(\mathbb{R} \backslash T)=0$. Выберем любое число $\delta \in\left(0, \min \left\{1,\left(\varepsilon^{\prime}-\varepsilon\right) / N\right\}\right)$. Для $t \in T$ обозначим $J_{1}(t)=$ $\left\{j: r_{\delta}\left(\mu[\cdot ; t], x_{j}(t)\right)>\varepsilon\right\}, J_{2}(t)=\{1, \ldots, N\} \backslash J_{1}(t)$. Если $j \in J_{1}(t)$, то $\mu\left[K\left(x_{j}(t), \varepsilon\right) ; t\right]<\delta$. Поэтому

$$
\begin{aligned}
\sum_{j \in J_{1}(t)} \lambda_{j}(t) & \leqslant \nu\left[\bigcup_{j \in J_{1}(t)} x_{j}(t) ; t\right] \\
& \leqslant \mu\left[\bigcup_{j \in J_{1}(t)} K\left(x_{j}(t), \varepsilon\right) ; t\right]+\varepsilon<N \delta+\varepsilon<\varepsilon^{\prime}
\end{aligned}
$$

В силу теоремы 4 найдутся функции $g_{j} \in S^{c}(\mathbb{R}, \mathscr{U}), j=1, \ldots, N$, такие, что $\operatorname{Mod} g_{j} \subset \operatorname{Mod} \mu[\cdot ; \cdot]+\operatorname{Mod} x_{j}(\cdot), g_{j}(t) \in \operatorname{supp} \mu[\cdot ; t]$ п.в. и $\rho\left(g_{j}(t), x_{j}(t)\right)<$ 
$r_{\delta}\left(\mu[\cdot ; t], x_{j}(t)\right)+\delta$ п.в. Используя неравенство (13), отсюда при п.в. $t \in \mathbb{R}$ получаем

$$
d\left(\sum_{j=1}^{N} \lambda_{j}(t) \delta_{g_{j}(t)}[\cdot], \nu[\cdot ; t]\right) \leqslant \max \left\{\sum_{j \in J_{1}(t)} \lambda_{j}(t), \max _{j \in J_{2}(t)} \rho\left(g_{j}(t), x_{j}(t)\right)\right\}<\varepsilon^{\prime}
$$

Лемма доказана.

Так как $\mu[\cdot ; \cdot] \in S^{c}\left(\mathbb{R}, \mathscr{M}_{\tau}\right)$, то (в силу теоремы 2) найдутся компактное множество $\mathscr{K} \subset \mathscr{M}_{\tau}$, множества $T_{l} \subset \mathbb{R}$ и меры $\mu_{l} \in \mathscr{M}_{\tau}, l \in \mathbb{N}$, такие, что $\left\{T_{l}\right\} \in \mathfrak{M}(\operatorname{Mod} \mu[\cdot ; \cdot]), \mu[\cdot ; t] \in \mathscr{K}$ при всех $t \in \bigcup_{l} T_{l}$ и $d\left(\mu[\cdot ; t], \mu_{l}[\cdot]\right)<\varepsilon / 10$ при всех $t \in T_{l}, l \in \mathbb{N}$. Сушествует конечная $\varepsilon / 10$-сеть для множества $\mathscr{K}$, состояшая из мер $\mu_{i}^{\prime} \in \mathscr{M}_{\tau}, i=1, \ldots, Q$. Каждому номеру $l \in \mathbb{N}$, для которого mes $T_{l} \neq 0$, можно поставить в соответствие какое-либо число $i=i(l) \in\{1, \ldots, Q\}$ так, что $d\left(\mu_{l}, \mu_{i(l)}^{\prime}\right)<\varepsilon / 5$. Обозначим

$$
\widetilde{T}_{i}=\bigcup_{\substack{l: \text { mes } T_{l} \neq 0 \\ i(l)=i}} T_{l}, \quad i=1, \ldots, Q
$$

Так как

$$
\lim _{m \rightarrow \infty} \sup _{\xi \in \mathbb{R}} \operatorname{mes}\left([\xi, \xi+1] \cap \bigcup_{l \geqslant m} T_{l}\right)=0,
$$

то $\left\{\widetilde{T}_{i}\right\} \in \mathfrak{M}(\operatorname{Mod} \mu[\cdot ; \cdot])$, функция $\mu[\cdot ; t]$ определена при всех $t \in \bigcup_{i} \widetilde{T}_{i}$ и $d\left(\mu[\cdot ; t], \mu_{i}^{\prime}[\cdot]\right)<3 \varepsilon / 10$ при всех $t \in \widetilde{T}_{i}, i=1, \ldots, Q$. Множество $\mathscr{M}_{\delta}$ плотно в метрическом пространстве $\left(\mathscr{M}_{\tau}, d\right)$, поэтому для каждого $i \in\{1, \ldots, Q\}$ найдется мера

$$
\nu_{i}=\sum_{k=1}^{n(i)} \lambda_{k}^{(i)} \delta_{x_{k}^{(i)}} \in \mathscr{M}_{\delta}
$$

такая, что $d\left(\mu_{i}^{\prime}, \nu_{i}\right)<\varepsilon / 10$, при этом, добавляя в случае необходимости к суммам, определяющим меры $\nu_{i}$, слагаемые, для которых $\lambda_{k}^{(i)}=0$ (и $x_{k}^{(i)}=x_{0}$ ), можно считать, что $n(i)=n \in \mathbb{N}$ для всех $i=1, \ldots, Q$. Положим

$$
\lambda_{k}(\cdot)=\sum_{i=1}^{Q} \lambda_{k}^{(i)} \chi_{\widetilde{T}_{i}}(\cdot), \quad x_{k}(\cdot)=\sum_{i=1}^{Q} x_{k}^{(i)} \chi_{\widetilde{T}_{i}}(\cdot), \quad k=1, \ldots, n .
$$

В силу леммы 2 имеем $\lambda_{k}(\cdot) \in S(\mathbb{R}, \mathbb{R}), \operatorname{Mod} \lambda_{k}(\cdot) \subset \operatorname{Mod} \mu[\cdot ; \cdot]$ и $x_{k}(\cdot) \in$ $S(\mathbb{R}, \mathscr{U}), \operatorname{Mod} x_{k}(\cdot) \subset \operatorname{Mod} \mu[\cdot ; \cdot]$. Так как функции $x_{k}(\cdot)$ принимают конечное число значений, то $x_{k}(\cdot) \in S^{c}(\mathbb{R}, \mathscr{U})$. Обозначим

$$
\nu[\cdot ; t]=\sum_{k=1}^{n} \lambda_{k}(t) \delta_{x_{k}(t)}[\cdot], \quad t \in \mathbb{R} .
$$


Тогда $d(\mu[\cdot ; t], \nu[\cdot ; t])<2 \varepsilon / 5$ при всех $t \in \bigcup_{i} \widetilde{T}_{i}$. Из леммы 9 следует существование функций $g_{k}(\cdot) \in S^{c}(\mathbb{R}, \mathscr{U}), k=1, \ldots, n$, таких, что $\operatorname{Mod} g_{k} \subset \operatorname{Mod} \mu[\cdot ; \cdot]$, $g_{k}(t) \in \operatorname{supp} \mu[\cdot ; t]$ п.в. и

$$
d\left(\nu[\cdot ; t], \sum_{k=1}^{n} \lambda_{k}(t) \delta_{g_{k}(t)}[\cdot]\right)<\frac{\varepsilon}{2}
$$

п.в. Найдутся числа $N \in \mathbb{N}$ и $m_{k}^{(i)} \in \mathbb{N}, i=1, \ldots, Q, k=1, \ldots, n$, такие, что $\sum_{k=1}^{n} m_{k}^{(i)}=N$ для всех $i$ и

$$
\left|\lambda_{k}^{(i)}-\frac{1}{N} m_{k}^{(i)}\right|<\frac{\varepsilon}{10 n}
$$

для всех $i$ и $k$. Для всех $i \in\{1, \ldots, Q\}$ и $j \in\{1, \ldots, N\}$ можно подобрать числа $k(i, j) \in\{1, \ldots, n\}$ так, чтобы равенство $k(i, j)=k$ при всех $i \in\{1, \ldots, Q\}$ и $k \in\{1, \ldots, n\}$ выполнялось ровно для $m_{k}^{(i)}$ чисел $j=\{1, \ldots, N\}$. Положим

$$
f_{j}(\cdot)=\sum_{i=1}^{Q} g_{k(i, j)}(\cdot) \chi_{\widetilde{T}_{i}}(\cdot), \quad j=1, \ldots, N
$$

Используя лемму 2, получаем $f_{j} \in S^{c}(\mathbb{R}, \mathscr{U})$ и $\operatorname{Mod} f_{j} \subset \operatorname{Mod} \mu[\cdot ; \cdot]$. При этом $f_{j}(t) \in \operatorname{supp} \mu[\cdot ; t]$ п.в. и

$$
\frac{1}{N} \sum_{j=1}^{N} \delta_{f_{j}(t)}[\cdot]=\frac{1}{N} \sum_{k=1}^{n} m_{k}^{(i)} \delta_{g_{k}(t)}[\cdot]
$$

при п.в. $t \in \widetilde{T}_{i}, i=1, \ldots, Q$. Отсюда (при п.в. $t \in \widetilde{T}_{i}, i=1, \ldots, Q$ ) следует неравенство

$$
\begin{aligned}
d\left(\mu[\cdot ; t], \frac{1}{N} \sum_{j=1}^{N} \delta_{f_{j}(t)}[\cdot]\right) \leqslant & d(\mu[\cdot ; t], \nu[\cdot ; t])+d\left(\nu[\cdot ; t], \sum_{k=1}^{n} \lambda_{k}(t) \delta_{g_{k}(t)}[\cdot]\right) \\
& +d\left(\sum_{k=1}^{n} \lambda_{k}(t) \delta_{g_{k}(t)}[\cdot], \frac{1}{N} \sum_{j=1}^{N} \delta_{f_{j}(t)}[\cdot]\right) \\
< & \frac{2 \varepsilon}{5}+\frac{\varepsilon}{2}+\sum_{k=1}^{n}\left|\lambda_{k}^{(i)}-\frac{1}{N} m_{k}^{(i)}\right|<\varepsilon
\end{aligned}
$$

Теорема 8 доказана. 


\section{Список литературы}

1. Былов Б.Ф., Виноград Р.Э., Лин В.Я., Локуииевский О.О. О топологических причинах аномального поведения некоторых почти периодических систем // Проблемы асимптотической теории нелинейных колебаний. Киев: Наукова думка, 1977. С. 54-61.

2. Данилов Л. И. Почти периодические сечения многозначных отображений // Известия отдела математики и информатики УдГУ. Вьп. 1. Ижевск, 1993. С. 16-78.

3. Долбилов A. М., Шнейберг И.Я. Почти периодические многозначные отображения и их сечения // Сиб. матем. журн. 1991. Т. 32. № 2. С. 172-175.

4. Castaing Ch., Valadier M. Convex analysis and measurable multifunctions // Lecture Notes in Math. V. 580. Berlin: Springer-Verlag, 1977.

5. Левин В. Л. Выпуклый анализ в пространствах измеримых функций и его применение в математике и экономике. М.: Наука, 1985.

6. Данилов Л.И. О сечениях многозначных почти периодических отображений // Деп. в ВИНИТИ 31.07 .95 , № 2340-В95.

7. Данилов Л. И. Многозначные почти периодические отображения и их сечения // Деп. в ВИНИТИ 24.09.93, № 2465-В93.

8. Данилов Л.И. О мерозначных почти периодических функциях // Вестн. Удмуртского ун-та. 1993. № 1. С. 51-58.

9. Данилов Л.И. О многозначных почти периодических отображениях, зависящих от параметра // Вестн. Удмуртского ун-та. 1994. № 2. С. 29-44.

10. Левитан Б. М. Почти-периодические функции. М.: Гостехиздат, 1953.

11. Левитан Б. М., Жиков В.В. Почти периодические функции и диффференциальные уравнения. М.: Изд-во МГУ, 1978.

12. Вахания Н. Н., Тариеладзе В.И., Чобанян С. А. Вероятностные распределения в банаховых пространствах. М.: Наука, 1985.

13. Данилов Л.И. О суперпозиции почти периодических многозначнњтх отображений и функций // Деп. в ВИНИТИ 31.01.95, № 262-В95.

14. Fryszkowski A. Continuous selections for a class of nonconvex multivalued maps // Studia Math. 1983. V. 76. P. 163-174.

15. Люстерник Л. А., Соболев В. И. Краткий курс функционалњного анализа. М.: Высшая школа, 1982.

16. Данилов Л.И., Иванов А.Г. К теореме о поточечном максимуме в почти периодическом случае // Изв. вузов. Сер. матем. 1994. №6. С. 50-59.

17. Данилов Л.И. О почти периодических мерозначных функциях // Деп. в ВИНИТИ 05.05.96, № 1434-B96. 